\title{
Hochschild Cohomology and Deformations of Clifford-Weyl Algebras
}

\author{
Ian M. MUSSON ${ }^{\dagger}$, Georges PINCZON ${ }^{\ddagger}$ and Rosane USHIROBIRA $\ddagger$ \\ $\dagger$ Department of Mathematical Sciences, University of Wisconsin-Milwaukee, \\ Milwaukee, WI 53201-0413, USA \\ E-mail: musson@uwm.edu \\ URL: http://www . uwm.edu/ musson/ \\ ¥ Institut de Mathématiques de Bourgogne, Université de Bourgogne, \\ B.P. 47870, F-21078 Dijon Cedex, France \\ E-mail: Georges.Pinczon@u-bourgogne.fr, Rosane.Ushirobira@u-bourgogne.fr \\ URL: http://www.u-bourgogne.fr/monge/phy.math/members/pinczon.htm, \\ http://www.u-bourgogne.fr/rosane.ushirobira
}

Received October 01, 2008, in final form February 25, 2009; Published online March 07, 2009 doi:10.3842/SIGMA.2009.028

\begin{abstract}
We give a complete study of the Clifford-Weyl algebra $\mathcal{C}(n, 2 k)$ from BoseFermi statistics, including Hochschild cohomology (with coefficients in itself). We show that $\mathcal{C}(n, 2 k)$ is rigid when $n$ is even or when $k \neq 1$. We find all non-trivial deformations of $\mathcal{C}(2 n+1,2)$ and study their representations.
\end{abstract}

Key words: Hochschild cohomology; deformation theory; Clifford algebras; Weyl algebras; Clifford-Weyl algebras; parastatistics

2000 Mathematics Subject Classification: 16E40; 16G99; 16S80; 17B56; 17B10; 53D55

\section{Introduction}

Throughout the paper, the base field is $\mathbb{C}$. As usual in superalgebra theory, we denote the ring $\mathbb{Z} / 2 \mathbb{Z}$ by $\mathbb{Z}_{2}$.

Let $\mathcal{C}(n)$ be the Clifford algebra with $n$ generators and $\mathrm{W}_{2 k}$ be the Weyl algebra with $2 k$ generators. Denote by $V_{\overline{0}}$ the vector space spanned by the generators of $\mathcal{C}(n)$. Elements of $V_{\overline{0}}$ will be called Fermi-type operators. Similarly, let $V_{\overline{1}}$ be the vector space spanned by the generators of $\mathrm{W}_{2 k}$. Elements of $V_{\overline{1}}$ will be called Bose-type operators.

There exist $\mathbb{Z}_{2}$-gradations on $\mathcal{C}(n)$ and $\mathrm{W}_{2 k}$ such that Fermi and Bose-type operators all have degree one. The Clifford-Weyl algebra is:

$$
\mathcal{C}(n, 2 k):=\mathcal{C}(n) \otimes_{\mathbb{Z}_{2}} \mathrm{~W}_{2 k},
$$

where $\otimes_{\mathbb{Z}_{2}}$ is relative to these gradations. It unifies Fermi and Bose-type operators in a unique algebra: as elements of $\mathcal{C}(n, 2 k)$, they anti-commute. There is a $\mathbb{Z}_{2}$-gradation on $\mathcal{C}(n, 2 k)$ extending the natural gradation of $V=V_{\overline{0}} \oplus V_{\overline{1}}$, and a corresponding structure of Lie superalgebra. Palev has shown that $V$ generates a sub-superalgebra of $\mathcal{C}(n, 2 k)$ isomorphic to $\mathfrak{o s p}(n+1,2 k)$, and introduced corresponding parastatistics relations [18]. This was an outcome of previous results by Wigner [28], Green [12] and others (see [8]). It gives an elegant algebraic interpretation of the Green ansatz, using the Hopf structure of the enveloping algebra of $\mathfrak{o s p}(n+1,2 k)$ [19], and introduces a construction of representations of parastatistics relations by Verma modules

\footnotetext{
${ }^{\star}$ This paper is a contribution to the Special Issue on Deformation Quantization. The full collection is available at http://www.emis.de/journals/SIGMA/Deformation_Quantization.html
} 
of $\mathfrak{o s p}(n+1,2 k)$. It also gives an idea of what deformed (quantum) parastatistics could be: replace $\mathcal{C}(n, 2 k)$ by a "quantum deformation", which still has a Hopf structure. This idea was developed by Palev himself [19] and other authors.

The purpose of the present paper is to return to the first steps of these theories: Clifford-Weyl algebras. At this level, there is a very natural question: does there exist non-trivial deformations of $\mathcal{C}(n, 2 k)$ ? By a deformation, we mean a formal one, in the sense of Gerstenhaber theory [11]. It is well-known that the answer is no for $\mathcal{C}(n, 0)=\mathcal{C}(n)$ and $\mathcal{C}(0,2 k)=\mathrm{W}_{2 k}$, but nothing was done in the general case. We shall answer the question, but this is not our only goal. We also want to introduce Clifford-Weyl algebras in a deformation quantization framework, emphasize their periodicity behavior and how it can be used, explain where Palev's theorem comes from, and so on.

Before describing the content of the paper, let us answer the initial question: $\mathcal{C}(2 n, 2 k)$ is rigid, for all $n, k, \mathcal{C}(2 n+1,2 k)$ is rigid if, and only if, $k \neq 1$, so the answer is no in these cases. In the case of $\mathcal{C}(2 n+1,2)$ there exist non-trivial deformations, that we completely describe in the paper, including their representations.

Let us give some details of our main results. In Section 1, we recall well-known properties of Clifford and Weyl algebras needed in the paper. In particular, we recall the deformation quantization construction of the Weyl algebra (resp. Clifford algebra) through the Moyal product (resp. a Moyal-type product).

In Section 2, we construct the Clifford-Weyl algebra $\mathcal{C}(n, 2 k)$ by a similar deformation quantization procedure, as a deformation of the super-exterior algebra of the $\mathbb{Z}_{2}$-graded vector space $V=V_{\overline{0}} \oplus V_{\overline{1}}$ with an explicit Moyal-type formula for the $\star$-product. From this construction, $\mathcal{C}(n, 2 k)$ is a $\mathbb{Z}_{2} \times \mathbb{Z}_{2}$-graded algebra, with natural left and right $\mathbb{Z}_{2}$-gradations.

We show in Section 3, that Clifford-Weyl algebras have a periodic behavior, very similar to the well-known, and useful, periodicity of Clifford algebras:

\section{Periodicity Lemma 1.}

$$
\mathcal{C}(2 m+n, 2 k) \simeq \mathcal{C}(2 m) \otimes \mathcal{C}(n, 2 k) .
$$

This simple Lemma has many consequences. For a given $r \in \mathbb{N}^{*}$, denote by $\mathcal{M}_{r}(A)$ the $r \times r$-matrix algebra with coefficients in an algebra $A$. One has:

\section{Theorem 1.}

1. $\mathcal{C}(2 n, 2 k) \simeq \mathcal{M}_{2^{n}}\left(\mathrm{~W}_{2 k}\right)$.

2. $\mathcal{C}(2 n+1,2 k) \simeq \mathcal{M}_{2^{n}}(\mathcal{C}(1,2 k))$.

Note that $\mathcal{C}(1,2 k)$ is the algebra generated by $\mathrm{W}_{2 k}$ and the parity operator of the metaplectic (oscillator) representation. In other words, $\mathcal{C}(1,2 k)$ is the smash product $\mathfrak{S}_{2} \ltimes \mathbf{W}_{2 k}$ where $\mathfrak{S}_{2}$ is the group $\{-1,1\}$ (see Remark 3.4). By Periodicity Lemma 1, it results that, though $\mathcal{C}(2 n, 2 k)$ has been defined by anti-commuting Fermi and Bose-type operators, sitting in osp $(2 n+1,2 k)$, it can also be defined by a new set of commuting generators, also of Fermi and Bose-type, no longer sitting in $\mathfrak{o s p}(2 n+1,2 k)$, but generating a superalgebra of type $\mathfrak{o}(2 n+1) \times \mathfrak{o s p}(1,2 k)$. This suggest that the enveloping algebra $\mathcal{U}(\mathfrak{o}(2 n+1) \times \mathfrak{o s p}(1,2 k))$ could be used for parastatistics. We shall not go further into this point in the present paper.

A second consequence, and the key for our purpose (to deform Clifford-Weyl algebras) is the natural Morita equivalence (see [14]) between $W_{2 k}$ and $\mathcal{C}(2 n, 2 k)$ (resp. $\mathcal{C}(1,2 k)$ and $\mathcal{C}(2 n+1,2 k))$. We shall see some consequences in Sections 5-8.

A new proof of Palev's theorem is given in Section 4:

Theorem 2 ([18]). The sub-superalgebra of $\mathcal{C}(n, 2 k)$ generated by Fermi and Bose-type operators is isomorphic to $\mathfrak{o s p}(n+1,2 k)$. 
The original proof uses a direct comparison of commutation rules, and does not really explain why the result exists. This is what we want to do, and the reason for giving this new proof. We explain it in a few words. First, we remark that $\mathcal{C}(n, 2 k)$ is $\mathbb{Z}_{2} \times \mathbb{Z}_{2}$-graded, and that the superbracket used in the Theorem 2 is the one defined by the right $\mathbb{Z}_{2}$-gradation. There exists a twisted adjoint action $\operatorname{ad}^{\prime}$ of $\mathcal{C}(n, 2 k)$ on itself, coming from the left $\mathbb{Z}_{2}$-gradation, and a supersymmetric bilinear form of type $(n+1,2 k)$ on $H:=\mathbb{C} \oplus V$ (graded by $H_{\overline{0}}=\mathbb{C} \oplus V_{\overline{0}}$, $\left.H_{\overline{1}}=V_{\overline{1}}\right)$ coming from the natural super-Poisson bracket. Then $\mathfrak{g}=V \oplus[V, V]$ is a subsuperalgebra of $\mathcal{C}(n, 2 k), H$ is $\operatorname{ad}^{\prime}(\mathfrak{g})$-stable, the bilinear form is $\operatorname{ad}^{\prime}(\mathfrak{g})$-invariant, and the result follows.

In Section 5, we go back to the initial question: to deform $\mathcal{C}(n, 2 k)$. As well-known in deformation theory, the first step is to study the Hochschild cohomology of $\mathcal{C}(n, 2 k)$ with coefficients in itself (see Appendix A for generalities in Hochschild cohomology and its relations with deformation theory). Since Morita equivalent algebras have isomorphic Hochschild cohomology [14], using the Periodicity Lemma 1, it results that:

\section{Theorem 3.}

1. $H^{\ell}(\mathcal{C}(2 n, 2 k))=\{0\}$ if $\ell>0$.

2. $H^{\ell}(\mathcal{C}(2 n+1,2 k))=H^{\ell}(\mathcal{C}(1,2 k))$, for all $\ell$.

So we have a partial answer to our question: $\mathcal{C}(2 n, 2 k)$ cannot be non-trivially deformed, and we are left with the case of $\mathcal{C}(1,2 k)$. As we mentioned before, $\mathcal{C}(1,2 k)$ is a smash product $\mathfrak{S}_{2} \ltimes \mathrm{W}_{2 k}$, but the cohomology of $\mathfrak{S}_{2} \ltimes \mathrm{W}_{2 k}$ is known, as a particular case of general results in [1] and [21], that give the cohomology of $G \ltimes \mathrm{W}_{2 k}$, when $G$ is a finite subgroup of $\mathrm{SP}(2 k)$. We obtain:

\section{Theorem 4.}

1. If $\ell>0$ and $\ell \neq 2 k$, then

$$
H^{\ell}(\mathcal{C}(2 n+1,2 k))=\{0\}
$$

2. $\operatorname{dim}\left(H^{2 k}(\mathcal{C}(2 n+1,2 k))\right)=1$. Denoting by $\omega_{1}, \ldots, \omega_{2 k+1}$ the basic Fermi-type operators and by $s$ the canonical symplectic form on $V_{\overline{1}}$, then there exists a $2 k$-cocycle $\Omega$ such that $H^{2 k}(\mathcal{C}(2 n+1,2 k))=\mathbb{C} \Omega$,

$$
\begin{aligned}
& \Omega\left(X_{1}, \ldots, X_{2 k}\right)=s^{k}\left(X_{1} \wedge \cdots \wedge X_{2 k}\right) \omega_{1} \cdots \omega_{2 k+1}, \quad \text { for } \quad X_{i} \in V_{\overline{1}}, \\
& \Omega\left(X_{1}, \ldots, X_{2 k}\right)=0, \quad \text { if one } \quad X_{i} \in V_{\overline{0}} .
\end{aligned}
$$

If a $2 k$-cocycle $\Omega^{\prime}$ verifies $\Omega^{\prime}\left(X_{1}, \ldots, X_{2 k}\right)=\Omega\left(X_{1}, \ldots, X_{2 k}\right)$ for all $X_{i} \in V$, then $\Omega^{\prime}=\Omega$ $\bmod B^{2 k}$.

As a consequence, $\mathcal{C}(2 n+1,2 k)$ is rigid if $k \neq 1$. In the case of $\mathcal{C}(2 n+1,2)$, since $H^{3}(\mathcal{C}(2 n+1,2))$ $=\{0\}$, there does exist non-trivial deformations, and more precisely, a universal deformation formula (see Appendix B).

Theorem 5. Let $\mathcal{A}_{\Lambda}$ be the $\mathbb{C}[\Lambda]$-algebra generated by $V_{\overline{0}}=\mathbb{C} P$ and $V_{\overline{1}}=\operatorname{span}\left\{E_{+}, E_{-}\right\}$with relations:

$$
\left[E_{+}, E_{-}\right]_{\mathcal{L}}=-\frac{1}{4}+\Lambda P, \quad P^{2}=1 \quad \text { and } \quad P E_{ \pm}=-E_{ \pm} P .
$$

Then $\mathcal{A}_{\Lambda}$ is a non-trivial polynomial deformation of $\mathcal{C}(1,2)$ and a universal deformation formula.

This algebra $\mathcal{A}_{\Lambda}$ is a particular case (the simplest one) of a symplectic reflection algebra [7]. 
Given an algebra $A$, any deformation $A_{\Lambda}$ of $A$ naturally produces a deformation $\mathcal{M}_{r}\left(A_{\Lambda}\right)$ of $\mathcal{M}_{r}(A)$ and conversely, any deformation of $\mathcal{M}_{r}(A)$ is of type $\mathcal{M}_{r}\left(A_{\Lambda}\right)$ up to equivalence (see Appendix C). Therefore, Theorem 1 allows us to conclude that $\mathcal{M}_{2^{n}}\left(\mathcal{A}_{\Lambda}\right)$ is a universal deformation formula of $\mathcal{C}(2 n+1,2)$.

Another presentation of this deformation is given in Section 6. We introduce algebras $\mathcal{A}_{\Lambda}(n)$ and $\mathcal{A}_{\lambda}(n)$ ( $\Lambda$ formal, $\lambda \in \mathbb{C}, n \in \mathbb{N}$ ) by generators and relations, with $\mathcal{A}_{\Lambda}(0)=\mathcal{A}_{\Lambda}$. We show that they can be constructed using Ore extensions, and that they have a periodic behavior:

Periodicity Lemma 2. $\mathcal{A}_{\Lambda}(n) \simeq \mathcal{C}(2 n) \otimes \mathcal{A}_{\Lambda} \simeq \mathcal{M}_{2^{n}}\left(\mathcal{A}_{\Lambda}\right)$ and the same result holds for $\mathcal{A}_{\lambda}$.

In Sections 7 and 8, we describe $\mathcal{A}_{\Lambda}(n)$ and $\mathcal{A}_{\lambda}(n)$ using the enveloping algebra $\mathcal{U}$ of the Lie superalgebra $\mathfrak{o s p}(1,2)$ and its primitive quotients [20]. Denoting by $\mathcal{A}_{\lambda}$ the algebra $\mathcal{A}_{\lambda}(0)$, one has:

\section{Theorem 6.}

1. $\mathcal{A}_{\Lambda}(n) \simeq \mathcal{M}_{2^{n}}\left(\mathfrak{S}_{2} \ltimes \mathcal{U}\right)$ and $\mathcal{A}_{\lambda}(n) \simeq \mathcal{A}_{\Lambda}(n) /(\Lambda-\lambda) \mathcal{A}_{\Lambda}(n)$.

2. Let $C$ be the Casimir element of $\mathcal{U}$ and $\mathcal{B}_{c}=\mathcal{U} /(C-c) \mathcal{U}, c \in \mathbb{C}$. Then $\mathcal{A}_{\lambda} \simeq \mathcal{B}_{\lambda^{2}-\frac{1}{16}}$ if $\lambda \neq 0$ and $\mathcal{A}_{0}=\mathcal{C}(1,2)$.

3. If $\lambda^{2} \neq\left(h+\frac{1}{4}\right)^{2}, 2 h \in \mathbb{N}$, then $\mathcal{A}_{\lambda}(n)$ is a simple algebra.

If $\lambda^{2}=\left(h+\frac{1}{4}\right)^{2}, 2 h \in \mathbb{N}$, then $\mathcal{A}_{\lambda}(n)$ is a primitive algebra with a unique non-zero twosided ideal that is the kernel of an irreducible representation $\pi_{h}$ of dimension $2^{n}(4 h+1)$.

4. $\mathcal{A}_{\Lambda}(n)$ is a FCR-algebra. Any finite-dimensional representation of $\mathcal{A}_{\lambda}(n)$ is completely reducible and isotypical of type $\pi_{h}$, if $\lambda^{2}=\left(h+\frac{1}{4}\right)^{2}, 2 h \in \mathbb{N}$.

We obtain in this way all primitive quotients of $\mathcal{A}_{\Lambda}(n): 2^{2 n}(4 h+1)^{2}$-dimensional quotients $(2 h \in \mathbb{N})$ and infinite-dimensional ones: $\mathcal{A}_{\lambda}(n) \simeq \mathcal{B}_{\lambda^{2}-\frac{1}{16}}$ if $\lambda \neq 0$ and $\mathcal{A}_{0}(n)=\mathcal{C}(2 n+1,2)$.

The isomorphism $\mathcal{A}_{\Lambda}(n) \simeq \mathcal{M}_{2^{n}}\left(\mathfrak{S}_{2} \ltimes \mathcal{U}\right)$ is useful to construct representations. Remark that representations of $\mathfrak{S}_{2} \ltimes \mathcal{U}$ are merely graded representations of $\mathcal{U}$. Then, from a graded representation of $\mathfrak{o s p}(1,2)$ on a space $H$, one constructs a representation of $\mathcal{A}_{\Lambda}(n)$ on $H^{2^{n}}$. All irreducible finite-dimensional representations are obtained in this way: from irreducible $(4 h+1)$-dimensional $(2 h \in \mathbb{N})$ representations of $\mathfrak{o s p}(1,2)$, one obtains $\pi_{h}$. From the metaplectic (oscillator) representation of $\mathfrak{o s p}(1,2)$, natural infinite-dimensional representations can be also obtained, using Dunkl-type formulas given in [13].

Finally, we extend the obtained supersymmetry by showing that $\mathcal{A}_{\Lambda}$ is a quotient of $\mathfrak{S}_{2} \ltimes$ $\mathcal{U}(\mathfrak{o s p}(2,2))$. Therefore $\mathcal{A}_{\Lambda}(n)$ is a quotient of $\mathcal{M}_{2^{n}}\left(\mathfrak{S}_{2} \ltimes \mathcal{U}(\mathfrak{o s p}(2,2))\right.$.

There are three Appendices: the first one is a short introduction to Hochschild cohomology relating it to deformation theory. In the second Appendix, we explain, with proofs, what a "universal deformation formula" is. We show in the third Appendix, that given an algebra $A$, deformations of $\mathcal{M}_{r}(A)$ are of type $\mathcal{M}_{r}\left(A_{\Lambda}\right)$ up to equivalence, where $A_{\Lambda}$ is a deformation of $A$. Results in Appendices B and $\mathrm{C}$ are known, but since we have not found a convenient reference, short proofs are given.

\section{Clifford algebras and Weyl algebras}

We begin by recalling some classical properties of Clifford and Weyl algebras needed in the paper. Throughout this section, we denote by $[\cdot, \cdot]$ the super bracket and by $[\cdot, \cdot]_{\mathcal{L}}$ the Lie bracket. 


\subsection{Weyl algebras}

Let $k \in \mathbb{N}^{*}$ and $\mathrm{S}_{2 k}=\mathbb{C}\left[p_{1}, q_{1}, \ldots, p_{k}, q_{k}\right]$ be the polynomial algebra in $2 k$ indeterminates equipped with the Poisson bracket:

$$
\{F, G\}=\sum_{i=1}^{k}\left(\frac{\partial F}{\partial p_{i}} \frac{\partial G}{\partial q_{i}}-\frac{\partial F}{\partial q_{i}} \frac{\partial G}{\partial p_{i}}\right), \quad \forall F, G \in \mathrm{S}_{2 k} .
$$

Let $\wp: \mathrm{S}_{2 k} \otimes \mathrm{S}_{2 k} \rightarrow \mathrm{S}_{2 k} \otimes \mathrm{S}_{2 k}$ be the operator defined by:

$$
\wp:=\sum_{i=1}^{k}\left(\frac{\partial}{\partial p_{i}} \otimes \frac{\partial}{\partial q_{i}}-\frac{\partial}{\partial q_{i}} \otimes \frac{\partial}{\partial p_{i}}\right) .
$$

Let $m$ be the product of $\mathrm{S}_{2 k}$ and $t$ be a formal parameter (or $t \in \mathbb{C}$ ). A new associative product $m_{\star}$ is defined by:

$$
m_{\substack{\star \\ t}}:=m \circ \exp \left(\frac{t}{2} \wp\right) .
$$

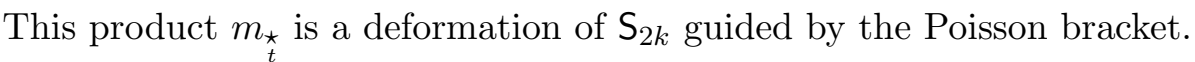

Definition 1.1. The Weyl algebra $\mathrm{W}_{2 k}$ is the vector space $\mathrm{S}_{2 k}$ endowed with the product $\star:=m_{\star}$, called the Moyal product.

A presentation of $\mathrm{W}_{2 k}$ is given by generators $\left\{p_{1}, q_{1}, \ldots, p_{k}, q_{k}\right\}$ and relations:

$$
[u, v]_{\mathcal{L}}=\{u, v\} \cdot 1, \quad \forall u, v \in \operatorname{span}\left\{p_{1}, q_{1}, \ldots, p_{k}, q_{k}\right\}
$$

Structurally, $\mathrm{W}_{2 k}$ is central, simple, naturally $\mathbb{Z}_{2}$-graded by the parity of $\mathrm{S}_{2 k}$ and has a supertrace [23]:

$$
\operatorname{Str}(F):=F(0), \quad \forall F \in \mathrm{W}_{2 k} .
$$

The space $\mathrm{M}_{k}:=\mathbb{C}\left[x_{1}, \ldots, x_{k}\right]$ is a faithful simple $\mathrm{W}_{2 k}$-module if we realize $p_{i}$ as $\frac{\partial}{\partial x_{i}}$ and $q_{i}$ as the multiplication by $x_{i}$, for all $i=1, \ldots, k$. In the sequel, $\mathrm{M}_{k}$ is called the metaplectic representation of the Weyl algebra $\mathrm{W}_{2 k}$.

The algebra of operators $\mathcal{L}\left(\mathrm{M}_{k}\right)$ appears as a completion of the Weyl algebra: $\mathrm{W}_{2 k}$ is the algebra of differential operators of finite order, and any element $T$ in $\mathcal{L}\left(\mathrm{M}_{k}\right)$ is a differential operator, in general of infinite order (i.e., in the formal sense, the sum is not finite), given by the formula:

$$
T=\sum_{N} \frac{1}{N !}\left(m \circ(T \otimes \mathcal{S}) \circ \Delta\left(x^{N}\right)\right) \frac{\partial^{N}}{\partial x^{N}},
$$

where $\mathcal{S}$ is the antipode of $\mathrm{M}_{2 k}, \Delta$ is its co-product, $x^{N}:=x_{1}^{n_{1}} \cdots x_{k}^{n_{k}}$ and $\frac{\partial^{N}}{\partial x^{N}}:=\frac{\partial^{n_{1}+\cdots+n_{k}}}{\partial x_{1}^{n_{1} \cdots \partial x_{k}^{n_{k}}}}$ if $N=\left(n_{1}, \ldots, n_{k}\right)$. This formula gives the (formal) symbol of $T$ in the normal ordering, and for well-behaved $T$, its (formal) symbol in the Weyl ordering (see [23]).

From the point of view of deformation theory, $\mathrm{W}_{2 k}$ is rigid. More precisely, we have $H^{r}\left(\mathrm{~W}_{2 k}\right)$ $=\{0\}$, for all $r>0[26]$.

We refer to [23] for more details on the Weyl algebra in the context of this section. 


\subsection{Clifford algebras}

Let $n \in \mathbb{N}^{*}$ and $\bigwedge_{n}$ be the Grassmann algebra in $n$ anti-commutative variables $\omega_{1}, \ldots, \omega_{n}$. Recall that $\bigwedge_{n}$ is $\mathbb{Z}$-graded. Denote by $\partial_{1}, \ldots, \partial_{n}$ the super-derivations defined by $\partial_{i}\left(\omega_{j}\right)=\delta_{i j}, \forall i, j$. The algebra $\bigwedge_{n}$ is endowed with a super Poisson bracket:

$$
\left\{\Omega, \Omega^{\prime}\right\}=2(-1)^{\operatorname{deg}_{\mathbb{Z}}(\Omega)+1} \sum_{i=1}^{n} \partial_{i}(\Omega) \wedge \partial_{i}\left(\Omega^{\prime}\right),
$$

for all $\Omega, \Omega^{\prime} \in \bigwedge_{n}[22]$. We define the operator $\wp$ of $\bigwedge_{n} \otimes \bigwedge_{n}$ by:

$$
\wp:=\sum_{i=1}^{n} \partial_{i} \otimes \partial_{i}
$$

where $\otimes$ is the graded tensor product of operators.

Let $m_{\wedge}$ be the product of $\bigwedge_{n}$ and $t$ be a formal parameter (or $t \in \mathbb{C}$ ). A new product $m_{\star}$ can be defined by (see [22]):

$$
m_{\star}:=m_{\wedge} \circ \exp (-t \wp) .
$$

Definition 1.2. The Clifford algebra $\mathcal{C}(n)$ is the vector space $\bigwedge_{n}$ equipped with the product $\star:=m_{\star}$.

There is a $\mathbb{Z}_{2}$-gradation on $\mathcal{C}(n)$ defined by $\operatorname{deg}_{\mathbb{Z}_{2}}\left(\omega_{i}\right)=1$, for all $i=1, \ldots, n$. A presentation of $\mathcal{C}(n)$ is given by basic generators $\omega_{1}, \ldots, \omega_{n}$ and relations:

$$
\left[v, v^{\prime}\right]=\left\{v, v^{\prime}\right\} \cdot 1, \quad \forall v, v^{\prime} \in \operatorname{span}\left\{\omega_{1}, \ldots, \omega_{n}\right\} .
$$

In particular, we have:

$$
\begin{aligned}
& \omega_{i}^{2}=1, \quad \forall i, \quad \omega_{i} \star \omega_{j}+\omega_{j} \star \omega_{i}=0, \quad \forall i \neq j \quad \text { and } \\
& \omega_{i_{1}} \wedge \cdots \wedge \omega_{i_{p}}=\omega_{i_{1}} \star \cdots \star \omega_{i_{p}} \quad \text { if } \quad i_{1}<i_{2}<\cdots<i_{p}, \quad p \leq n .
\end{aligned}
$$

\subsection{Even Clifford algebras}

For $i=1, \ldots, n$, let $P_{i}=\partial_{i}$ and $Q_{i}=x_{i} \wedge$. be respectively the operator of derivation and multiplication of the Grassmann algebra $\Phi_{n}$ in $n$ anti-commutative variables $x_{1}, \ldots, x_{n}$. The operators $\omega_{2 j-1}=Q_{j}+P_{j}$ and $\omega_{2 j}=i\left(Q_{j}-P_{j}\right), j=1, \ldots, n$ verify the defining relations of the Clifford algebra $\mathcal{C}(2 n)$, so there is a homomorphism from $\mathcal{C}(2 n)$ onto the algebra of differential operators $\operatorname{Diff}\left(\Phi_{n}\right)$. It is easy to see that $\operatorname{dim}(\mathcal{C}(2 n))=\operatorname{dim}\left(\operatorname{Diff}\left(\Phi_{n}\right)\right)=\operatorname{dim}\left(\mathcal{L}\left(\Phi_{n}\right)\right)$, so we can identify $\mathcal{C}(2 n)=\operatorname{Diff}\left(\Phi_{n}\right)=\mathcal{M}_{2^{n}}(\mathbb{C})$, where $\mathcal{M}_{2^{n}}(\mathbb{C})$ denotes the algebra of complex matrices of order $2^{n}$. As a consequence, $\Phi_{n}$ is the unique simple $\mathcal{C}(2 n)$-module, called the spin representation of $\mathcal{C}(2 n)$.

Structurally, the even Clifford algebra $\mathcal{C}(2 n)=\mathcal{M}_{2^{n}}(\mathbb{C})$ is simple and its center is $\mathbb{C}$. From the point of view of deformation theory, $\mathcal{C}(2 n)$ is rigid and we have $H^{r}(\mathcal{C}(2 n))=\{0\}$ if $r>0$.

Since $\mathcal{C}(2 n)=\mathcal{M}_{2^{n}}(\mathbb{C})$, there is a natural trace on $\mathcal{C}(2 n)$ that can be written in an analogous way as in (2):

$$
\operatorname{Tr}(\Omega):=2^{n} \Omega(0), \quad \forall \Omega \in \mathcal{C}(2 n) .
$$

There is also a similar formula to $(3)$ in the case of $\mathcal{C}(2 n)$. In other words, any operator $T \in \mathcal{L}\left(\Phi_{n}\right)$ is differential and an explicit formula is given by:

$$
T=\sum_{I \in\{0,1\}^{n}}(-1)^{\theta(I, I)}\left(m_{\wedge} \circ(T \otimes \mathcal{S}) \circ \Delta\left(x^{I}\right)\right) \wedge \partial^{I},
$$


where $\theta$ is the bilinear form on $\mathbb{N}^{n}$ associated to the matrix $\left(\theta_{r s}\right)_{r, s=1}^{n}$ with $\theta_{r s}=1$ if $r>s$ and 0 otherwise, $\otimes$ is the non-graded tensor product of operators, $\mathcal{S}$ is the antipode of $\Phi_{n}, \Delta$ is its co-product, $x^{I}:=x_{1}^{i_{1}} \wedge \cdots \wedge x_{n}^{i_{n}}$ and $\partial^{I}:=\partial_{1}^{i_{1}} \circ \cdots \circ \partial_{n}^{i_{n}}$ if $I=\left(i_{1}, \ldots, i_{n}\right)$.

\subsection{Periodicity of Clifford algebras}

There is an algebra isomorphism between $\mathcal{C}(2 n+k)$ and $\mathcal{C}(2 n) \otimes \mathcal{C}(k)$ since $\omega_{1} \otimes 1, \ldots, \omega_{2 n} \otimes 1$ and $i^{n} \omega_{1} \star \cdots \star \omega_{2 n} \otimes \omega_{j}^{\prime}, j=1, \ldots, k$ in $\mathcal{C}(2 n) \otimes \mathcal{C}(k)$ verify the defining relations of $\mathcal{C}(2 n+k)$ thanks to the formula:

$$
\left(\omega_{1} \star \cdots \star \omega_{2 n}\right)^{2}=(-1)^{n} .
$$

It results that:

$$
\mathcal{C}(2 n) \simeq \mathcal{C}(2)^{\otimes_{n}} \quad \text { and } \quad \mathcal{C}(2 n+1) \simeq \mathcal{C}(2 n) \otimes \mathcal{C}(1) \simeq \mathcal{M}_{2^{n}}(\mathcal{C}(1))
$$

\subsection{Odd Clifford algebras}

Since $\mathcal{C}(1) \simeq \mathbb{C} \times \mathbb{C}$, from the isomorphism $\mathcal{C}(2 n+1) \simeq \mathcal{M}_{2^{n}}(\mathcal{C}(1))$ it follows that $\mathcal{C}(2 n+1)$ is the product $\mathcal{C}(2 n) \times \mathcal{C}(2 n)$. Therefore $H^{r}(\mathcal{C}(2 n+1))=\{0\}$ if $r>0$, and that implies that $\mathcal{C}(2 n+1)$ is rigid.

We will make more explicit the above isomorphism: $\mathcal{C}(2 n+1) \simeq \mathcal{C}(2 n) \times \mathcal{C}(2 n)$. The element $z=\omega_{1} \star \cdots \star \omega_{2 n+1}$ is central and verifies $z^{2}=(-1)^{n}$. Set $Z=\operatorname{span}\{1, z\}$. Then $\mathcal{C}(2 n+1) \simeq$ $Z \otimes \mathcal{C}(2 n)$ as algebras. Let $z_{+}=\frac{1}{2}\left(1+i^{n} z\right)$ and $z_{-}=\frac{1}{2}\left(1-i^{n} z\right)$. Therefore $z_{ \pm}^{2}=z_{ \pm}$, $z_{+} \star z_{-}=z_{-} \star z_{+}=0$ and $1=z_{+}+z_{-}$. We conclude that

$$
\mathcal{C}(2 n+1)=z_{+} \star \mathcal{C}(2 n) \oplus z_{-} \star \mathcal{C}(2 n),
$$

that is, a reduction of $\mathcal{C}(2 n+1)$ in a direct sum of two ideals isomorphic to $\mathcal{C}(2 n)$ as algebras. It follows that $\mathcal{C}(2 n+1)$ has exactly two $2^{n}$-dimensional simple modules built from the spin representation of $\mathcal{C}(2 n)$. To give more details, we need the following lemma:

Lemma 1.3. Let $P$ be the natural parity of $\Phi_{n}$. Then in the spin representation of $\mathcal{C}(2 n)$, one has:

$$
\omega_{1} \star \cdots \star \omega_{2 n}=i^{n} P \text {. }
$$

Proof. We set $T=\omega_{1} \star \cdots \star \omega_{2 n}$. The operator $T$ of $\Phi_{n}$ is diagonalizable since $T^{2}=(-1)^{n}$, and it commutes with $P$. We denote by $S_{\overline{0}, \pm i^{n}}$ and $S_{\overline{1}, \pm i^{n}}$ its eigenspaces in $S_{\overline{0}}$ and $S_{\overline{1}}$ respectively, where $S=\Phi_{n}$. The subspaces $S_{\overline{0}, i^{n}} \oplus S_{\overline{1},-i^{n}}$ and $S_{\overline{0},-i^{n}} \oplus S_{\overline{1}, i^{n}}$ are $\mathcal{C}(2 n)$-stable since $T$ anticommutes with $\omega_{i}, 1 \leq i \leq 2 n$. It follows that $T= \pm i^{n} P$. To determine the sign, we compute:

$$
T(1)=i^{n}\left(Q_{1}+P_{1}\right) \star\left(Q_{2}-P_{2}\right) \star \cdots \star\left(Q_{n}+P_{n}\right) \star\left(Q_{n}-P_{n}\right)(1)=i^{n} .
$$

Finally, we obtain $T=i^{n} P$.

The element $z$ is central, $z^{2}=(-1)^{n}$, hence $z= \pm i^{n}$ in any simple $\mathcal{C}(2 n+1)$-module. Since $z=\left(\omega_{1} \star \cdots \star \omega_{2 n}\right) \star \omega_{2 n+1}$, using the lemma we obtain the spin representations $\Phi_{n}^{ \pm}$of $\mathcal{C}(2 n+1)$ as follows: $\mathcal{C}(2 n) \subset \mathcal{C}(2 n+1)$ acts on $\Phi_{n}$ by the spin representation (see Section 1.3), for $\Phi_{n}^{+}$, define $\omega_{2 n+1}=P$ and for $\Phi_{n}^{-}$, define $\omega_{2 n+1}=-P$. 


\subsection{Weyl algebras and supersymmetry}

Let $\mathbf{W}=\mathbf{W}_{2 k}=\oplus_{r \geq 0} \mathbf{W}^{r}$ be the $\mathbb{Z}$-gradation of the vector space $\mathbf{W}$. Recall that $\mathbf{W}$ is a $\mathbb{Z}_{2}$-graded algebra. By (1), we have:

$$
[F, G]_{\mathcal{L}}=\{F, G\}, \quad \forall F \in \bigoplus_{r \leq 2} \mathrm{~W}^{r} .
$$

Let $\mathfrak{h}=\mathfrak{h}_{\overline{1}} \oplus \mathfrak{h}_{\overline{0}}$ where $\mathfrak{h}_{\overline{1}}=\mathrm{W}^{1}=\operatorname{span}\left\{p_{i}, q_{i}, i=1, \ldots, k\right\}$ and $\mathfrak{h}_{\overline{0}}=\mathrm{W}^{2}$. The super bracket stabilizes $\mathfrak{h}$. Besides, $\mathfrak{h}$ is isomorphic to the Lie superalgebra $\mathfrak{o s p}(1,2 k)$. In particular, $\mathfrak{h}_{\overline{0}} \simeq \mathfrak{s p}(2 k)$ and the adjoint action of $\mathfrak{h}_{\overline{0}}$ on $\mathfrak{h}_{\overline{1}}$ is the standard action of $\mathfrak{s p}(2 k)$ on $\mathbb{C}^{2 k}$. As a consequence, $\mathrm{W}$ is a semisimple $\mathfrak{h}_{\overline{0}}$-module for the adjoint action and $\mathbf{W}=\oplus_{r \geq 0} \mathrm{~W}^{r}$ is its reduction in isotypical components.

By (1), we have:

$$
[v, F]=2 v F, \quad \forall F \in \mathrm{W}^{2 r+1} \quad \text { and } \quad[v, F]_{\mathcal{L}}=\{v, F\}, \quad \forall F \in \mathrm{W}^{2 r}, \quad v \in \mathrm{W}^{1} .
$$

Therefore $W$ is also semi-simple for the adjoint action of $\mathfrak{h}$ and $W=\oplus_{r \geq 0} A^{r}$ is its reduction into isotypical components, where $A_{0}=\mathbb{C}$ and $\mathrm{A}^{r}=\mathrm{W}^{2 r-1} \oplus \mathrm{W}^{2 r}$, if $r>0$. We refer to [17] or [23] for more details.

\subsection{Clifford algebras and symmetry}

Let $\mathcal{C}=\mathcal{C}(n)$. There is a $\mathbb{Z}$-gradation on the vector space $\mathcal{C}$ and, as an algebra, $\mathcal{C}$ is $\mathbb{Z}_{2}$-graded. By (4), we have:

$$
\left[\Omega, \Omega^{\prime}\right]=\left\{\Omega, \Omega^{\prime}\right\}, \quad \forall \Omega \in \bigoplus_{r \leq 2} \mathcal{C}^{r} .
$$

Let $\mathfrak{g}=\mathfrak{g}_{\overline{1}} \oplus \mathfrak{g}_{\overline{0}}$ where $\mathfrak{g}_{\overline{1}}=\mathcal{C}^{1}$ and $\mathfrak{g}_{\overline{0}}=\mathcal{C}^{2}$. The Lie bracket stabilizes $\mathfrak{g}$. Moreover, $\mathfrak{g}$ is isomorphic to the Lie algebra $\mathfrak{o}(n+1)$. In particular, $\mathfrak{g}_{\overline{0}} \simeq \mathfrak{o}(n)$ and the adjoint action of $\mathfrak{g}_{\overline{0}}$ on $\mathfrak{g}_{\overline{1}}$ is the standard action of $\mathfrak{o}(n)$ on $\mathbb{C}^{n}$. The direct sum $\mathfrak{g}=\mathfrak{g}_{\overline{1}} \oplus \mathfrak{g}_{\overline{0}}$ is a $\mathbb{Z}_{2}$-gradation for the Lie algebra $\mathfrak{g}$, that is $\left[\mathfrak{g}_{i}, \mathfrak{g}_{j}\right]_{\mathcal{L}} \subset \mathfrak{g}_{i+j}$ (this is not a graded Lie algebra!). For the adjoint action, $\mathcal{C}$ is a semisimple $\mathfrak{g}_{\overline{0}}$-module and is isomorphic to the $\mathfrak{o}(n)$-module $\bigwedge \mathbb{C}^{n}$, whose reduction into isotypical components is well-known (see [24] or [9]). The reduction into isotypical components of the $\mathfrak{g}$-module $\mathcal{C}$ can be deduced, but this is simply not the subject of this paper.

\section{Clifford-Weyl algebras}

We recall the construction of the exterior algebra of a $\mathbb{Z}_{2}$-graded vector space $V=V_{\overline{0}} \oplus V_{\overline{1}}$ : let $\bigwedge:=\bigwedge V_{\overline{0}}$ be the exterior algebra of $V_{\overline{0}}$ and $\mathrm{S}:=\operatorname{Sym}\left(V_{\overline{1}}\right)$ be the symmetric algebra of $V_{\overline{1}}$. Using their $\mathbb{Z}$-gradation, define a $\mathbb{Z} \times \mathbb{Z}_{2}$ gradation on $\Lambda$ and on $S$ by

$$
\bigwedge^{(i, \overline{0})}=\bigwedge^{i}, \quad \bigwedge^{(i, \overline{1})}=\{0\} \quad \text { and } \quad \mathrm{S}^{(i, \bar{i})}=\mathrm{S}^{i}, \quad \mathrm{~S}^{(i, \bar{j})}=\{0\} \quad \text { if } \quad \bar{i} \neq \bar{j} .
$$

The exterior algebra of $V$ is the $\mathbb{Z} \times \mathbb{Z}_{2}$-graded algebra

$$
\mathcal{E}:=\bigwedge_{\mathbb{Z} \times \mathbb{Z}_{2}}^{\otimes} \mathrm{S}=\bigwedge_{\mathbb{Z}}^{\otimes} \mathrm{S}
$$

endowed with the product:

$$
(\Omega \otimes F) \wedge\left(\Omega^{\prime} \otimes F^{\prime}\right)=(-1)^{f \omega^{\prime}}\left(\Omega \wedge \Omega^{\prime}\right) \otimes F F^{\prime},
$$


for all $\Omega \in \bigwedge, \Omega^{\prime} \in \bigwedge^{\omega^{\prime}}, F \in \mathrm{S}^{f}, F^{\prime} \in \mathrm{S}$. We have

$$
A^{\prime} \wedge A=(-1)^{a a^{\prime}+b b^{\prime}} A \wedge A^{\prime}, \quad \forall A \in \mathcal{E}^{(a, \bar{b})}, \quad A^{\prime} \in \mathcal{E}^{\left(a^{\prime}, \overline{b^{\prime}}\right)}
$$

and that means that $\mathcal{E}$ is $\mathbb{Z} \times \mathbb{Z}_{2}$-commutative.

Now, assume that the dimension of $V_{\overline{1}}$ is even, say $\operatorname{dim}\left(V_{\overline{1}}\right)=2 k$. Set $n=\operatorname{dim}\left(V_{\overline{0}}\right)$.

We have defined Poisson brackets on $\bigwedge$ and $S$ in Section 1. Associated operators $\wp_{\wedge}$ and $\wp_{\mathrm{S}}$ are respectively defined on $\Lambda \otimes \wedge$ and $S \otimes S$.

A super $\mathbb{Z} \times \mathbb{Z}_{2}$-Poisson bracket on $\mathcal{E}$ is then defined by:

$$
\left\{\Omega \otimes F, \Omega^{\prime} \otimes F^{\prime}\right\}=(-1)^{f \omega^{\prime}}\left(\left\{\Omega, \Omega^{\prime}\right\} \otimes F F^{\prime}+\left(\Omega \wedge \Omega^{\prime}\right) \otimes\left\{F, F^{\prime}\right\}\right),
$$

for all $\Omega \in \Lambda, \Omega^{\prime} \in \bigwedge^{\omega^{\prime}}, F \in \mathrm{S}^{f}, F^{\prime} \in \mathrm{S}$. Now, let $\sigma_{23}$ and $\wp$ be operators on $\mathcal{E} \otimes \mathcal{E}$ defined by:

$$
\begin{aligned}
& \sigma_{23}\left(\Omega \otimes F \otimes \Omega^{\prime} \otimes F^{\prime}\right)=(-1)^{f \omega^{\prime}} \Omega \otimes \Omega^{\prime} \otimes F \otimes F^{\prime}, \\
& \wp=\sigma_{23} \circ\left(-2 \wp_{\wedge} \otimes \mathrm{Id}+\mathrm{Id} \otimes \wp_{\mathrm{s}}\right) \circ \sigma_{23},
\end{aligned}
$$

for all $\Omega \in \bigwedge, \Omega^{\prime} \in \bigwedge^{\omega^{\prime}}, F \in \mathrm{S}^{f}, F^{\prime} \in \mathrm{S}$.

Let $t$ be a formal parameter (or $t \in \mathbb{C}$ ). A new product $m_{\star}$ on $\mathcal{E}$ is defined from these operators and from the product $m_{\mathcal{E}}$ on $\mathcal{E}$ by:

$$
m_{\star}:=m_{\mathcal{E}} \circ \exp \left(\frac{t}{2} \wp\right) .
$$

Since $m_{\star}=m_{\wedge} \otimes m_{\mathrm{S}} \circ \exp \left(-t \wp_{\wedge}\right) \otimes \exp \left(\frac{t}{2} \wp_{\mathrm{S}}\right) \circ \sigma_{23}$, it results that $m_{\star}$ is exactly the $\mathbb{Z}_{2} \times \mathbb{Z}_{2^{-}}$ graded tensor algebra product:

$$
\mathcal{C}^{t}(n) \otimes_{\mathbb{Z}_{2} \times \mathbb{Z}_{2}} \mathrm{~W}_{2 k}^{t}=\mathcal{C}^{t}(n) \otimes_{\mathbb{Z}_{2}} \mathrm{~W}_{2 k}^{t},
$$

where $\otimes_{\mathbb{Z}_{2}}$ means the graded tensor product with respect to left $\mathbb{Z}_{2}$-gradations, $\mathcal{C}(n)^{t}$ denotes the algebra equipped with product $m_{\star}$ and similarly for $\mathbf{W}_{2 k}^{t}$ (see Section 1 ). By definition, $m_{\star}$ is a deformation of $m_{\mathcal{E}}$ guided by the Poisson super bracket.

Definition 2.1. The Clifford-Weyl algebra $\mathcal{C}(n, 2 k)$ is the vector space $\mathcal{E}$ endowed with the product $\star:=m_{\star}$.

Denote by $\left\{\omega_{1}, \ldots, \omega_{n}\right\}$ and $\left\{p_{1}, q_{1}, \ldots, p_{k}, q_{k}\right\}$ respectively the basis of $\mathcal{C}(n)$ and $\mathrm{W}_{2 k}$ as in Section 1 . The algebra $\mathcal{C}(n, 2 k)$ has a presentation given by generators $\left\{\omega_{1}, \ldots, \omega_{n}, p_{1}, q_{1}, \ldots\right.$, $\left.p_{k}, q_{k}\right\}$ and relations

$$
\begin{aligned}
& {\left[\omega_{i}, \omega_{j}\right]_{+}=2 \delta_{i j}, \quad\left[p_{i}, q_{j}\right]_{-}=\delta_{i j}, \quad\left[p_{i}, p_{j}\right]_{-}=\left[q_{i}, q_{j}\right]_{-}=0 \quad \text { if } \quad i \neq j} \\
& \text { and }\left[\omega_{i}, p_{j}\right]_{+}=0, \quad\left[\omega_{i}, q_{j}\right]_{+}=0, \quad \forall i, j,
\end{aligned}
$$

where $[A, B]_{ \pm}:=A \star B \pm B \star A$.

When $n=2 \ell$ is even, we set

$$
P_{j}=\frac{1}{2}\left(\omega_{2 j-1}+i \omega_{2 j}\right) \quad \text { and } \quad Q_{j}=\frac{1}{2}\left(\omega_{2 j-1}-i \omega_{2 j}\right) \quad \text { for } \quad j \leq \ell .
$$

The first two relations above become:

$$
\left[P_{i}, Q_{j}\right]_{+}=\delta_{i j}, \quad\left[P_{i}, P_{j}\right]_{+}=\left[Q_{i}, Q_{j}\right]_{+}=0 .
$$

Consider now $\Phi_{\ell}$ the Grassmann algebra in $\ell$ anti-commutative variables $\xi_{1}, \ldots, \xi_{\ell}, \mathrm{M}_{k}$ the polynomial algebra in $k$ variables $x_{1}, \ldots, x_{k}$ and $\operatorname{SM}(\ell, k)$ the exterior algebra of the $\mathbb{Z}_{2}$-graded 
space $W=W_{\overline{0}} \oplus W_{\overline{1}}$ with $W_{\overline{0}}=\operatorname{span}\left\{\xi_{1}, \ldots, \xi_{\ell}\right\}$ and $W_{\overline{1}}=\operatorname{span}\left\{x_{1}, \ldots, x_{k}\right\}$. There is a $\mathcal{C}(2 \ell, 2 k)$ module structure on $\operatorname{SM}(\ell, k)$ given by $P_{i}=\frac{\partial}{\partial \xi_{i}}, Q_{i}=\xi_{i} \wedge \cdot(1 \leq i \leq \ell), p_{j}=\frac{\partial}{\partial x_{j}}, q_{j}=x_{j}$. $(1 \leq j \leq k)$. Besides, $\operatorname{SM}(\ell, k)$ is a simple $\mathcal{C}(2 \ell, 2 k)$-module. In the sequel, we call $\operatorname{SM}(\ell, k)$ the spin-metaplectic representation of $\mathcal{C}(n, 2 k)$. This provides a homomorphism from $\mathcal{C}(2 \ell, 2 k)$ onto the algebra of differential operators of the $\mathbb{Z}_{2} \times \mathbb{Z}_{2}$-graded exterior algebra $\operatorname{SM}(\ell, k)$. We will show later that $\mathcal{C}(n, 2 k)$ is simple, so we have actually an isomorphism, $\mathcal{C}(2 \ell, 2 k) \simeq \operatorname{Diff}(\operatorname{SM}(\ell, k))$ and that generalizes the cases of $\mathcal{C}(2 \ell)$ and $W_{2 k}$ seen in Section 1.

When $n=2 \ell+1$ is odd, we obtain two spin-metaplectic representations $\operatorname{SM}(\ell, k)^{ \pm}$of $\mathcal{C}(2 \ell+1$, $2 k$ ) by acting $\mathcal{C}(2 \ell, 2 k)$ on $\operatorname{SM}(\ell, k)$ as above and by setting $\omega_{2 \ell+1}=Q$ for $\operatorname{SM}(\ell, k)^{+}$and $\omega_{2 \ell+1}=-Q$ for $\operatorname{SM}(\ell, k)^{-}$, where $Q$ is the parity:

$$
Q(\omega \otimes f)=(-1)^{\operatorname{deg}_{\mathbb{Z}}(\omega)+\operatorname{deg}_{\mathbb{Z}}(f)} \omega \otimes f, \quad \forall \omega \in \Phi_{\ell}, \quad f \in \mathrm{M}_{k} .
$$

It will be shown later that if $k \neq 0, \mathcal{C}(2 \ell+1,2 k)$ is simple and as a consequence, both representations $\operatorname{SM}(\ell, k)^{ \pm}$are faithful.

\section{Periodicity of Clifford-Weyl algebras}

Clifford algebras have a periodic behavior Section 1.4 and we now show that this periodicity can be extended to Clifford-Weyl algebras. We denote by $\mathcal{C}(r)$, the Clifford algebra in $r$ variables and by $\mathrm{W}_{2 k}$, the Weyl algebra constructed from $2 k$ variables (see Section 1 ).

\section{Lemma 3.1 (Periodicity Lemma 1).}

$$
\mathcal{C}(2 m+n, 2 k) \simeq \mathcal{C}(2 m) \otimes \mathcal{C}(n, 2 k)
$$

Proof. Let $\left\{\omega_{1}, \ldots, \omega_{2 m}\right\}$ and $\left\{\omega_{1}^{\prime}, \ldots, \omega_{n}^{\prime}, p_{1}, q_{1}, \ldots, p_{k}, q_{k}\right\}$ be respectively the set of generators of $\mathcal{C}(2 m)$ and $\mathcal{C}(n, 2 k)$. Let $z=i^{m} \omega_{1} \star \cdots \star \omega_{2 m}$. So $z^{2}=1$ and $z$ anti-commutes with $\omega_{1}, \ldots, \omega_{2 m}$. The following elements of $\mathcal{C}(2 m) \otimes \mathcal{C}(n, 2 k): \omega_{1} \otimes 1, \ldots, \omega_{2 m} \otimes 1, z \otimes \omega_{1}^{\prime}, \ldots, z \otimes \omega_{n}^{\prime}$, $z \otimes p_{1}, \ldots, z \otimes p_{k}$ and $z \otimes q_{1}, \ldots, z \otimes q_{k}$ verify the defining relations of $\mathcal{C}(2 m+n, 2 k)$. Since they generate $\mathcal{C}(2 m) \otimes \mathcal{C}(2 n, k)$ as an algebra, we get an algebra homomorphism from $\mathcal{C}(2 m+n, 2 k)$ onto $\mathcal{C}(2 m) \otimes \mathcal{C}(n, 2 k)$.

Denote by $\left\{\widetilde{\omega_{1}}, \ldots, \widetilde{\omega}_{2 m+n}, \widetilde{p_{1}}, \widetilde{q_{1}}, \ldots, \widetilde{p_{k}}, \widetilde{q_{k}}\right\}$ the set of generators of $\mathcal{C}(2 m+n, 2 k)$. Let $\widetilde{z}=i^{m} \widetilde{\omega_{1}} \star \cdots \star \widetilde{\omega}_{2 m}$. So $\widetilde{z}^{2}=1, \widetilde{z}$ anti-commutes with $\widetilde{\omega_{1}}, \ldots, \widetilde{\omega}_{2 m+n}$ and $\widetilde{z}$ commutes with

$p_{1}, \ldots, p_{k}$ and $q_{1}, \ldots, q_{k}$. The following elements of $\mathcal{C}(2 m+n, 2 k): \widetilde{\omega}_{1}, \ldots, \widetilde{\omega}_{2 m}, \widetilde{z} \star \widetilde{\omega}_{2 m+1}, \ldots$, $\widetilde{z} \star \widetilde{\omega}_{2 m+n}, \widetilde{z} \star \widetilde{p_{1}}, \widetilde{z} \star \widetilde{q_{1}}, \ldots, \widetilde{z} \star \widetilde{p_{k}}, \widetilde{z} \star \widetilde{q_{k}}$ verify the defining relations of $\mathcal{C}(2 m) \otimes \mathcal{C}(n, 2 k)$, so we get the inverse homomorphism.

Corollary 3.2. One has:

$$
\begin{aligned}
& \mathcal{C}(2 n, 2 k) \simeq \mathcal{C}(2 n) \otimes \mathrm{W}_{2 k} \simeq \mathcal{M}_{2^{n}}\left(\mathrm{~W}_{2 k}\right) \quad \text { and } \\
& \mathcal{C}(2 n+1,2 k) \simeq \mathcal{C}(2 n) \otimes \mathcal{C}(1,2 k) \simeq \mathcal{M}_{2^{n}}(\mathcal{C}(1,2 k))
\end{aligned}
$$

where $\mathcal{M}_{r}(A)$ denotes the $r \times r$-matrix algebra with coefficients in an algebra $A$ for a given $r \in \mathbb{N}^{*}$.

\section{Corollary 3.3.}

1. $\mathcal{C}(2 n, 2 k)$ is simple with center $\mathbb{C}$.

2. If $k \neq 0$, then $\mathcal{C}(2 n+1,2 k)$ is simple with center $\mathbb{C}$. 
Proof. 1. $\mathcal{C}(2 n, 2 k) \simeq \mathcal{M}_{2^{n}}\left(\mathrm{~W}_{2 k}\right)$ is simple since $\mathrm{W}_{2 k}$ is simple.

2. Since $\mathcal{C}(2 n+1,2 k) \simeq \mathcal{M}_{2^{n}}(\mathcal{C}(1,2 k))$, it is enough to prove the result for $\mathcal{C}(1,2 k)$.

But $\mathcal{C}(1,2 k) \simeq \mathfrak{S}_{2} \ltimes \mathbf{W}_{2 k}$ and $\mathbf{W}_{2 k}$ is simple, so the result is a particular case of a general theorem in [16].

For the sake of completeness, here is a direct proof: we write $\mathcal{C}(1,2 k)=\mathcal{C}(1) \underset{\mathbb{Z}_{2}}{\otimes \mathrm{W}_{2 k}}$ where $\mathcal{C}(1)$ is the Clifford algebra generated by $\mathcal{P}$ such that $\mathcal{P}^{2}=1$. Recall that using the Moyal $\star$-product, the Weyl algebra $\mathrm{W}=\mathrm{W}_{2 k}$ can be realized as a deformation of the polynomial algebra $\mathbb{C}\left[p_{1}, q_{1}, \ldots, p_{k}, q_{k}\right]$. Fix $p=p_{1}$ and $q=q_{1}$.

We have $[p, f]_{\mathcal{L}}=\frac{\partial f}{\partial q}, \forall f \in \mathrm{W}$. In addition, for all $g \in \mathrm{W}$ :

$$
\begin{aligned}
{[p, \mathcal{P} \star g]_{\mathcal{L}} } & =p \star \mathcal{P} \star g-\mathcal{P} \star g \star p=-\mathcal{P} \star(p \star g+g \star p) \\
& =-\mathcal{P} \star\left(p g+\frac{1}{2}\{p, g\}+g p+\frac{1}{2}\{g, p\}\right)=-2 \mathcal{P} \star(p g) .
\end{aligned}
$$

Let $I$ be a non-zero two-sided ideal of $\mathcal{C}(1,2 k)$ and let $f+\mathcal{P} \star g \in I, f+\mathcal{P} \star g \neq 0$. Then $[p, f+\mathcal{P} \star g]_{\mathcal{L}} \in I$ gives $\frac{\partial f}{\partial q}-2 \mathcal{P} \star(p g) \in I$ and we can reiterate. Hence:

- if $g=0$, then $f \in I$. It follows that $I \cap \mathrm{W} \neq\{0\}$;

- if $g \neq 0$, since there exists $j$ such that $\frac{\partial^{j} f}{\partial q^{j}}=0$, one has $(-1)^{j} 2^{j} \mathcal{P} \star\left(p^{j} g\right) \in I$, implying $p^{j} g \in I$. But $p^{j} g \neq 0$, so it follows that $I \cap \mathrm{W} \neq\{0\}$ as well.

In both cases, $I \cap \mathrm{W}$ is a non-zero ideal of the Weyl algebra $\mathrm{W}$. Since $\mathrm{W}$ is simple, $I \cap \mathrm{W}=\mathrm{W}$. So $1 \in I$ and we conclude that $I=\mathcal{C}(1,2 k)$.

The center of $\mathcal{C}(1,2 k)$ is $\mathbb{C}$ since the center of $\mathrm{W}$ is $\mathbb{C}$.

Remark 3.4. Let us first recall what a smash product is. Let $A$ be an algebra, $G$ a finite group acting on $A$ by automorphisms and $\mathbb{C}[G]$ the group algebra. The smash product $G \ltimes A$ is the algebra with underlying space $A \otimes \mathbb{C}[G]$ and product defined by:

$$
(a \otimes g)\left(a^{\prime} \otimes g^{\prime}\right)=a\left(g \cdot a^{\prime}\right) \otimes g g^{\prime}, \quad \forall a, a^{\prime} \in A, \quad g, g^{\prime} \in G .
$$

Smash products used in this paper are defined from a $\mathbb{Z}_{2}$-graded algebra $A$ and $G=\mathfrak{S}_{2}=$ $\{-1,1\}$. Denoting by $\mathcal{P}$ the parity operator of $A, \mathcal{P}(a):=(-1)^{\operatorname{deg}(a)} a, \forall a \in A, \mathfrak{S}_{2}$ acts on $A$ by $(-1)^{\alpha} \cdot a:=\mathcal{P}^{\alpha}(a), \forall a \in A, \alpha=0,1$ and there is a corresponding smash product $\mathfrak{S}_{2} \ltimes A$. It is the algebra generated by $\mathcal{P}$ and $A$ with relations $\mathcal{P} a=\mathcal{P}(a) \mathcal{P}, \forall a \in A$ and $\mathcal{P}^{2}=1$. It is easy to check that $\mathfrak{S}_{2} \ltimes A$-modules and $\mathbb{Z}_{2}$-graded $A$-modules are exactly the same notion.

Now consider the Clifford-Weyl algebra $\mathcal{C}(1,2 k)$. Using the $\mathbb{Z}_{2}$-graded structure of $\mathrm{W}_{2 k}$, $\mathcal{C}(1,2 k) \simeq \mathfrak{S}_{2} \ltimes \mathrm{W}_{2 k}$. Also $\mathcal{C}(1,2 k)$ is isomorphic to a subalgebra of $\mathcal{M}_{2}\left(\mathrm{~W}_{2 k}\right)$ :

$$
\mathcal{C}(1,2 k) \simeq\left\{\left(\begin{array}{cc}
a & b \\
\sigma(b) & \sigma(a)
\end{array}\right), a, b \in \mathrm{W}_{2 k}\right\}
$$

where $\sigma$ is the parity operator of $\mathbf{W}_{2 k}$. In this isomorphism, $\omega_{1} \in \mathcal{C}(1)$ is realized as the matrix $\left(\begin{array}{ll}0 & 1 \\ 1 & 0\end{array}\right)$ and $\mathrm{W}_{2 k}$ as $\left\{\left(\begin{array}{cc}a & 0 \\ 0 & \sigma(a)\end{array}\right), a \in \mathrm{W}_{2 k}\right\}$.

Finally, $\mathcal{C}(1,2 k)$ is isomorphic to the algebra generated by the parity operator $\mathcal{P}$ of $\mathrm{M}_{k}=$ $\mathbb{C}\left[x_{1}, \ldots, x_{k}\right]$ and $\mathbf{W}_{2 k}$, realized as the algebra of differential operators of $\mathbf{M}_{k}$ (see Section 1 ).

\section{Clifford-Weyl algebras and supersymmetry}

Let us consider the $\mathbb{Z}_{2} \times \mathbb{Z}_{2}$-graded algebra $\mathcal{C}(n, 2 k)$ and the subspace $V=V_{\overline{0}} \oplus V_{\overline{1}}$ where $V_{\overline{0}}=\mathcal{C}(n, 2 k)_{(\overline{1}, \overline{0})}=\bigwedge_{n}^{1}$ and $V_{\overline{1}}=\mathcal{C}(n, 2 k)_{(\overline{1}, \overline{1})}=\mathrm{S}_{2 k}^{1}$ (see Section 2 for the notation). 
If $k=0$, then $V_{\overline{0}} \oplus\left[V_{\overline{0}}, V_{\overline{0}}\right]_{\mathcal{L}}$ is a Lie algebra for the natural Lie bracket of the Clifford algebra, isomorphic to $\mathfrak{o}(n+1)$ and $\left[V_{\overline{0}}, V_{\overline{0}}\right]_{\mathcal{L}}$ is a Lie subalgebra isomorphic to $\mathfrak{o}(n)$ (for details, see Section 1).

If $n=0$, then $V_{\overline{1}} \oplus\left[V_{\overline{1}}, V_{\overline{1}}\right]$ is a Lie superalgebra for the natural super bracket of the Weyl algebra, isomorphic to $\mathfrak{o s p}(1,2 k)$ and $\left[V_{\overline{1}}, V_{\overline{1}}\right]$ is a Lie algebra isomorphic to $\mathfrak{s p}(2 k)$.

To generalize this situation, we need some notation: for an element $a \in \mathcal{C}(n, 2 k)$, denote its $\mathbb{Z}_{2} \times \mathbb{Z}_{2}$-degree by $\Delta(a):=\left(\Delta_{1}(a), \Delta_{2}(a)\right)$. We consider $\mathcal{C}(n, 2 k)$ as an algebra $\mathbb{Z}_{2}$-graded by $\Delta_{2}$ and we denote by $[\cdot, \cdot]$ the associated super bracket.

The proposition below shows how to realize $\mathfrak{o s p}(n+1,2 k)$ as a Lie sub-superalgebra of $\mathcal{C}(n, 2 k)$. This important result was first obtained by [18] for $\mathfrak{o s p}(2 \ell+1,2 k)$. We propose here another method to show the same result, inspired by [23] and based on a well-chosen twisted adjoint action.

Proposition 4.1 ([18]). Let $\mathfrak{g}=V \oplus[V, V]$. Then $\mathfrak{g}$ is a Lie sub-superalgebra of $\mathcal{C}(n, 2 k)$ isomorphic to $\mathfrak{o s p}(n+1,2 k)$. Moreover

$$
\mathfrak{g}_{\overline{0}}=V_{\overline{0}} \oplus\left[V_{\overline{0}}, V_{\overline{0}}\right] \oplus\left[V_{\overline{1}}, V_{\overline{1}}\right]
$$

with $\left[V_{\overline{0}}, V_{\overline{0}}\right] \simeq \mathfrak{o}(n),\left[V_{\overline{1}}, V_{\overline{1}}\right] \simeq \mathfrak{s p}(2 k), V_{\overline{0}} \oplus\left[V_{\overline{0}}, V_{\overline{0}}\right] \simeq \mathfrak{o}(n+1)$ and $\mathfrak{g}_{\overline{0}} \simeq \mathfrak{o}(n+1) \times \mathfrak{s p}(2 k)$. Also,

$$
\mathfrak{g}_{\overline{1}}=V_{\overline{1}} \oplus\left[V_{\overline{0}}, V_{\overline{1}}\right]
$$

and $V_{\overline{1}} \oplus\left[V_{\overline{1}}, V_{\overline{1}}\right] \simeq \mathfrak{o s p}(1,2 k)$. If we set $\mathfrak{h}=\left[V_{\overline{0}}, V_{\overline{0}}\right] \oplus\left[V_{\overline{1}}, V_{\overline{1}}\right] \oplus\left[V_{\overline{0}}, V_{\overline{1}}\right]$, then $\mathfrak{h} \simeq \mathfrak{o s p}(n, 2 k)$.

Proof. By a case by case straightforward computation, using the product formula (5), we get the formula:

$$
[[X, Y], Z]=2\left(\{Y, Z\} X-(-1)^{\Delta_{2}(X) \Delta_{2}(Y)}\{X, Z\} Y\right), \quad \forall X, Y, Z \in V,
$$

where $\{\cdot, \cdot\}$ is the super Poisson bracket defined in Section 2.

Hence $[[V, V], V] \subset V$. If $H \in[V, V]$ and $X, Y \in V$, then:

$$
[H,[X, Y]]=[[H, X], Y]+(-1)^{\Delta_{2}(H) \Delta_{2}(X)}[X,[H, Y]] .
$$

Using (6), we conclude that $[[V, V],[V, V]] \subset[V, V]$, therefore $\mathfrak{g}$ is a Lie superalgebra and $\mathfrak{h}$ is a sub-superalgebra.

To prove the isomorphisms, we set $V^{\prime}=\mathbb{C} \oplus V$. Define a non-degenerate supersymmetric 2 -form $(\cdot \mid \cdot)$ on $V^{\prime}$ by:

$$
(X \mid Y):=\{X, Y\}, \quad \forall X, Y \in V \quad \text { and } \quad(1 \mid 1)=-2 .
$$

Then formula (6) becomes parastatistics relations:

$$
[[X, Y], Z]=2\left((Y \mid Z) X-(-1)^{\Delta_{2}(X) \Delta_{2}(Y)}(X \mid Z) Y\right), \quad \forall X, Y, Z \in V .
$$

Next, we define the $\Delta_{1}$-twisted adjoint representation of the Lie superalgebra $\mathcal{C}(n, 2 k)$ :

$$
\operatorname{ad}^{\prime}(a)(b):=a \star b-(-1)^{\Delta_{2}(a) \Delta_{2}(b)+\Delta_{1}(a)} b \star a, \quad \forall a, b \in \mathcal{C}(n, 2 k) .
$$

It is easy to check that it is indeed a representation. If $H \in \mathfrak{h}, \operatorname{ad}^{\prime}(H)=\operatorname{ad}(H)$, writing $H=[X, Y]$ and using (PS), one obtains:

$$
\left(\operatorname{ad}^{\prime}(H)(Z) \mid T\right)=-(-1)^{\Delta_{2}(Z) \Delta_{2}(H)}\left(Z \mid \operatorname{ad}^{\prime}(H)(T)\right), \quad \forall T \in V,
$$


henceforth $\operatorname{ad}^{\prime}(\mathfrak{h})\left(V^{\prime}\right) \subset V^{\prime}$ and $\mathfrak{h} \subset \mathfrak{o s p}(n, 2 k)$. Since both spaces have the same dimension $\frac{n(n-1)}{2}+2 n k+k(2 k+1)($ see $[25])$, it follows $\mathfrak{h} \simeq \mathfrak{o} \mathfrak{s p}(n, 2 k)$.

It remains to examine the action of $\operatorname{ad}^{\prime}(X)$ on $V^{\prime}$ when $X \in V$. We have $\operatorname{ad}^{\prime}(X)(Y)=0$ if $X \in V_{\bar{i}}, Y \in V_{\bar{j}}$ with $\bar{i} \neq \bar{j}$. Moreover, if $X, Y \in V_{\overline{0}}$, then $\operatorname{ad}^{\prime}(X)(Y)=X \star Y+Y \star X=$ $\{X, Y\} \cdot 1=(X \mid Y)$. If $X, Y \in V_{\overline{1}}$, then $\operatorname{ad}^{\prime}(X)(Y)=X \star Y-Y \star X=\{X, Y\} \cdot 1=(X \mid Y)$. Since $\operatorname{ad}^{\prime}(X)(1)=2 X$, finally $\left(\operatorname{ad}^{\prime}(X)(Y) \mid 1\right)=-2(X \mid Y)=-(-1)^{\Delta_{2}(X) \Delta_{2}(Y)}\left(Y \mid \operatorname{ad}^{\prime}(X)(1)\right)$. So $\mathfrak{g} \subset \mathfrak{o s p}(n+1,2 k)$ and both spaces have the same dimension.

Corollary 4.2. Let $V=V_{\overline{0}} \oplus V_{\overline{1}}$ be a $\mathbb{Z}_{2}$-graded space with $\operatorname{dim}\left(V_{\overline{0}}\right)=n$ and $\operatorname{dim}\left(V_{\overline{1}}\right)=2 k$. Assume that $V$ is equipped with a non-degenerate supersymmetric bilinear form $(\cdot \mid \cdot)$. Let $A$ be the $\mathbb{Z}_{2}$-graded algebra generated by $V=V_{\overline{0}} \oplus V_{\overline{1}}$ and relations (PS). Then $A$ is isomorphic to the enveloping algebra $\mathcal{U}(\mathfrak{o s p}(n+1,2 k))$.

Proof. We denote by $[\cdot, \cdot]_{A}$ the super bracket of $A$. Proceeding exactly as in the proof of Proposition 4.1, we show that $V+[V, V]_{A}$ is a Lie superalgebra using the parastatistics relations (PS). From the definition of $A$ together with Proposition 4.1, there is an algebra homomorphism from $A$ onto $\mathcal{C}(n, 2 k)$ that is the identity when restricted to $V$. This homomorphism induces a Lie superalgebra homomorphism from $V+[V, V]_{A}$ onto $V \oplus[V, V]$ (realized in $\mathcal{C}(n, 2 k)$ and isomorphic to $\mathfrak{o s p}(n+1,2 k)$ by Proposition 4.1). That implies $\operatorname{dim}\left(V+[V, V]_{A}\right) \geq \operatorname{dim}(\mathfrak{o s p}(n+1,2 k))$.

On the other hand, $\operatorname{dim}\left(V+[V, V]_{A}\right) \leq \operatorname{dim}(V \oplus[V, V])$ since we can write

$$
[V, V]_{A}=\left[V_{\overline{0}}, V_{\overline{0}}\right]_{A}+\left[V_{\overline{1}}, V_{\overline{1}}\right]_{A}+\left[V_{\overline{0}}, V_{\overline{1}}\right]_{A}
$$

and $\operatorname{dim}([V, V])=\operatorname{dim}\left(V_{\overline{0}} \wedge V_{\overline{0}}\right)+\operatorname{dim}\left(V_{\overline{0}} \otimes V_{\overline{1}}\right)+\operatorname{dim}\left(V_{\overline{0}} V_{\overline{1}}\right)$.

It results that $V \oplus[V, V]_{A} \simeq \mathfrak{o s p}(n+1,2 k)$. Remark that the parastatistics relations hold in the enveloping algebra $\mathcal{U}(\mathfrak{o s p}(n+1,2 k))$ since they hold in $\mathfrak{o s p}(n+1,2 k)$. To finish, we apply the universal property of $\mathcal{U}(\mathfrak{o s p}(n+1,2 k))$.

Remark 4.3. The result in Proposition 4.1 is helpful to obtain explicit descriptions of $\mathfrak{o s p}(n+1$, $2 k$ ) (for instance, the root system).

Remark 4.4. As observed in [18], the fact that generators of $\mathcal{C}(n)$ (Fermi-type operators) and those of $\mathrm{W}_{2 k}$ (Bose-type operators) anti-commute in $\mathcal{C}(n, 2 k)$ is a main argument to prove that the Lie sub-superalgebra that they generate is $\mathfrak{o s p}(n+1,2 k)$. However, the periodicity of Clifford-Weyl algebras, namely $\mathcal{C}(2 n, 2 k) \simeq \mathcal{C}(2 n) \otimes \mathrm{W}_{2 k}$, shows that it is always possible to obtain $\mathcal{C}(2 n, 2 k)$ from commuting Bose-type and Fermi-type operators (that will not live in the Lie superalgebra $\mathfrak{o s p}(2 n+1,2 k)$, but rather in $\mathfrak{o}(2 n+1) \times \mathfrak{o s p}(1,2 k))$.

In the sequel, all $\star$ products will simply be denoted by juxtaposition.

\section{Cohomology of Clifford-Weyl algebras}

In Appendix A, the reader can find a short introduction to Hochschild cohomology of an algebra with coefficients in itself.

By Periodicity Lemma 1 and Corollary 3.2, we have

$$
\mathcal{C}(2 n, 2 k) \simeq \mathcal{M}_{2^{n}}\left(\mathrm{~W}_{2 k}\right) \quad \text { and } \quad \mathcal{C}(2 n+1,2 k) \simeq \mathcal{M}_{2^{n}}(\mathcal{C}(1,2 k)) .
$$

But for an algebra $A, \mathcal{M}_{r}(A)$ and $A$ have isomorphic cohomology spaces [14], so it results that the cohomology of Clifford-Weyl algebras can be computed from the cohomology of $\mathrm{W}_{2 k}$ and $\mathcal{C}(1,2 k)$ :

\section{Proposition 5.1.}

1. $H^{\ell}(\mathcal{C}(2 n, 2 k))=\{0\}$ if $\ell>0$.

2. $H^{\ell}(\mathcal{C}(2 n+1,2 k))=H^{\ell}(\mathcal{C}(1,2 k))$, for all $\ell$. 
Proof. It is enough to remark that $H^{\ell}\left(\mathrm{W}_{2 k}\right)=\{0\}$ if $\ell>0$ [26].

We now give more details on the identifications in the above Proposition. We use the isomorphisms in Corollary 3.2: $\mathcal{C}(2 n, 2 k) \simeq \mathcal{C}(2 n) \otimes \mathrm{W}_{2 k}$ and $\mathcal{C}(2 n+1,2 k) \simeq \mathcal{C}(2 n) \otimes \mathcal{C}(1,2 k)$. The letter $A$ denotes either $\mathrm{W}_{2 k}$ or $\mathcal{C}(1,2 k)$.

Since $\mathcal{C}(2 n)$ is separable, we compute the cohomology of $\mathcal{C}(2 n) \otimes A$ using normalized $\mathcal{C}(2 n)$ relative cochains (see [10]), that is, cochains

$$
\Omega:(\mathcal{C}(2 n) \otimes A)^{\ell} \rightarrow \mathcal{C}(2 n) \otimes A
$$

that verify:

$$
\begin{aligned}
& \Omega\left(C a_{1}, a_{2}, \ldots, a_{\ell}\right)=C \Omega\left(a_{1}, \ldots, a_{\ell}\right), \\
& \Omega\left(a_{1}, \ldots, a_{i} C, a_{i+1}, \ldots, a_{\ell}\right)=\Omega\left(a_{1}, \ldots, a_{i}, C a_{i+1}, \ldots, a_{\ell}\right), \\
& \Omega\left(a_{1}, \ldots, a_{\ell} C\right)=\Omega\left(a_{1}, \ldots, a_{\ell}\right) C, \\
& \Omega\left(a_{1}, \ldots, a_{\ell}\right)=0 \quad \text { if one } a_{i} \in \mathcal{C}(2 n)
\end{aligned}
$$

for all $C \in \mathcal{C}(2 n)$. Since $\mathcal{C}(2 n)$ commutes with $A$, such a cochain is completely determined by its restriction $\widetilde{\Omega}: A^{\ell} \rightarrow \mathcal{C}(2 n) \otimes A$ verifying

$$
C \widetilde{\Omega}\left(a_{1}, \ldots, a_{\ell}\right)=\widetilde{\Omega}\left(a_{1}, \ldots, a_{\ell}\right) C .
$$

for all $C \in \mathcal{C}(2 n)$. It results that $\widetilde{\Omega}$ is actually $A$-valued. Then the map $\Omega \rightsquigarrow \widetilde{\Omega}$ induces an isomorphism [10]:

$$
H^{\ell}(\mathcal{C}(2 n) \otimes A) \simeq H^{\ell}(A) .
$$

To obtain the desired cohomology, that is, $H^{\ell}(\mathcal{C}(2 n, 2 k))$ or $H^{\ell}(\mathcal{C}(2 n+1,2 k))$, we use the isomorphism $\phi: \mathcal{C}(2 n) \otimes A \rightarrow \mathcal{C}(2 n, 2 k)$ or $\mathcal{C}(2 n+1,2 k)$ in the Periodicity Lemma 1 (Lemma 3.1): given a cochain $\Omega$ of $\mathcal{C}(2 n) \otimes A$, we introduce a cochain $\phi^{*}(\Omega)$ of $\mathcal{C}(2 n, 2 k)$ or $\mathcal{C}(2 n+1,2 k)$ defined by

$$
\phi^{*}(\Omega)\left(x_{1}, \ldots, x_{\ell}\right)=\phi\left(\Omega\left(\phi^{-1}\left(x_{1}\right), \ldots, \phi^{-1}\left(x_{\ell}\right)\right)\right),
$$

for all $x_{1}, \ldots, x_{\ell} \in \mathcal{C}(2 n, 2 k)$ or $\mathcal{C}(2 n+1,2 k)$. Then the map $\Omega \rightsquigarrow \phi^{*}(\Omega)$ induces a cohomology isomorphism.

It remains to compute the cohomology of $\mathcal{C}(1,2 k)=\mathcal{C}(1) \otimes_{\mathbb{Z}_{2}} \mathrm{~W}_{2 k}$. Since $\mathcal{C}(1,2 k)=\mathfrak{S}_{2} \ltimes \mathrm{W}_{2 k}$, this is a particular case of a result in [1] where the cohomology of $G \ltimes \mathrm{W}_{2 k}$ is given for $G$ a finite group of symplectic linear transformations. There is an improved version of this result in [21], that allows a better management of cocycles. Denote by $P$ the generator of $\mathcal{C}(1)$ satisfying $P^{2}=1$. One has:

Proposition $5.2([1,21])$.

1. If $\ell>0$ and $\ell \neq 2 k$, then

$$
H^{\ell}(\mathcal{C}(1,2 k))=\{0\} .
$$

2. $\operatorname{dim}\left(H^{2 k}(\mathcal{C}(1,2 k))\right)=1$. Moreover, there exists a normalized $\mathcal{C}(1)$-relative cocycle $\theta$ such that $H^{2 k}(\mathcal{C}(1,2 k))=\mathbb{C} \theta$ and

$$
\theta\left(X_{1}, \ldots, X_{2 k}\right)=s^{k}\left(X_{1} \wedge \cdots \wedge X_{2 k}\right) P, \quad \text { for } \quad X_{1}, \ldots, X_{2 k} \in V_{\overline{1}},
$$

where $s$ is the canonical symplectic form on $V_{\overline{1}}$. If a $2 k$-cocycle $\theta^{\prime}$ verifies $\theta^{\prime}\left(X_{1}, \ldots, X_{2 k}\right)=$ $\theta\left(X_{1}, \ldots, X_{2 k}\right)$ for all $X_{i} \in V$, then $\theta^{\prime}=\theta \bmod B^{2 k}$. 
Proof. See [1] for the dimension of $H^{\ell}(\mathcal{C}(1,2 k))$. See [21] for the last claims.

\section{Corollary 5.3.}

1. If $\ell>0$ and $\ell \neq 2 k$, then

$$
H^{\ell}(\mathcal{C}(2 n+1,2 k))=\{0\} .
$$

2. Denote by $\omega_{1}, \ldots, \omega_{2 n}, P$ the canonical generators of $\mathcal{C}(2 n+1)$ realized in $\mathcal{C}(2 n+1,2 k)$. Then there exists a cocycle $\Omega$ such that such that $H^{2 k}(\mathcal{C}(2 n+1,2 k))=\mathbb{C} \Omega$,

$$
\begin{aligned}
& \Omega\left(X_{1}, \ldots, X_{2 k}\right)=i^{n} s^{k}\left(X_{1} \wedge \cdots \wedge X_{2 k}\right) \omega_{1} \ldots \omega_{2 k+1}, \quad \text { for } \quad X_{i} \in V_{\overline{1}}, \\
& \Omega\left(X_{1}, \ldots, X_{2 k}\right)=0, \quad \text { if one } X_{i} \in V_{\overline{0}} .
\end{aligned}
$$

If a $2 k$-cocycle $\Omega^{\prime}$ verifies $\Omega^{\prime}\left(X_{1}, \ldots, X_{2 k}\right)=\Omega\left(X_{1}, \ldots, X_{2 k}\right)$ for all $X_{i} \in V$, then $\Omega^{\prime}=\Omega$ $\bmod B^{2 k}$.

Proof. Proposition 5.2 provides a cocycle $\theta$ that allows us to construct a cocycle $\widehat{\theta}$ of $\mathcal{C}(2 n) \otimes$ $\mathcal{C}(1,2 k)$ such that:

$$
\widehat{\theta}\left(C_{1} \otimes x_{1}, \ldots, C_{2 k} \otimes x_{2 k}\right)=C_{1} \cdots C_{2 k} \otimes \theta\left(x_{1}, \ldots, x_{2 k}\right)
$$

for $x_{1}, \ldots, x_{2 k} \in \mathrm{W}_{2 k}, C_{1}, \ldots, C_{2 k} \in \mathcal{C}(2 n)$. Next we compute $\Omega=\phi^{*}(\widehat{\theta})$ using formulas in the proof of Lemma 3.1:

$$
\begin{aligned}
\Omega\left(X_{1}, \ldots, X_{2 k}\right) & =\phi\left(\widehat{\theta}\left(i^{n} \omega_{1} \cdots \omega_{2 n} \otimes X_{1}, \ldots, i^{n} \omega_{1} \cdots \omega_{2 n} \otimes X_{2 k}\right)\right) \\
& =\phi\left(\left(i^{n}\right)^{2 k}\left(\omega_{1} \cdots \omega_{2 n}\right)^{2 k} s^{k}\left(X_{1} \wedge \cdots \wedge X_{2 k}\right) P\right)
\end{aligned}
$$

for $X_{1}, \ldots, X_{2 k} \in V_{\overline{1}}$. Since $\left(\omega_{1} \cdots \omega_{2 n}\right)^{2}=(-1)^{n}$ (see Section 1), then

$$
\Omega\left(X_{1}, \ldots, X_{2 k}\right)=\phi\left(s^{k}\left(X_{1} \wedge \cdots \wedge X_{2 k}\right) P\right)=i^{n} s^{k}\left(X_{1} \wedge \cdots \wedge X_{2 k}\right) \omega_{1} \cdots \omega_{2 n} P .
$$

Corollary 5.4. The Clifford-Weyl algebra $\mathcal{C}(2 n+1,2 k)$ is rigid if $k \neq 1$.

Since $\operatorname{dim}\left(H^{2}(\mathcal{C}(2 n+1,2))\right)=1$ and $H^{3}(\mathcal{C}(2 n+1,2))=\{0\}$, then $\mathcal{C}(2 n+1,2)$ can be non trivially deformed by a universal deformation formula (see Appendix B). For $\mathcal{C}(1,2)$, this formula is a particular case of a symplectic reflection algebras (see [7]):

Proposition 5.5. Let $\mathcal{A}_{\Lambda}$ be the $\mathbb{C}[\Lambda]$-algebra generated by $V_{\overline{0}}=\mathbb{C} P$ and $V_{\overline{1}}=\operatorname{span}\left\{E_{+}, E_{-}\right\}$ with relations:

$$
\left[E_{+}, E_{-}\right]_{\mathcal{L}}=-\frac{1}{4}+\Lambda P, \quad P^{2}=1 \quad \text { and } \quad P E_{ \pm}=-E_{ \pm} P .
$$

Then $\mathcal{A}_{\Lambda}$ is a non-trivial polynomial deformation of $\mathcal{C}(1,2)$ and a universal deformation formula.

Proof. See [7] or [21].

\section{Universal deformation formula of $\mathcal{C}(2 n+1,2)$}

Definition 6.1. Let $\mathcal{A}_{\lambda}(n), \lambda \in \mathbb{C}$ be the algebra with generators $\omega_{1}, \ldots, \omega_{2 n+1}, E_{ \pm}$and relations:

$$
\begin{aligned}
& {\left[E_{+}, E_{-}\right]_{\mathcal{L}}=-\frac{1}{4}+i^{n} \lambda \omega_{1} \cdots \omega_{2 n+1},} \\
& \omega_{j} \omega_{k}+\omega_{k} \omega_{j}=2 \delta_{j k} \quad(1 \leq j, k \leq 2 n+1), \\
& E_{ \pm} \omega_{j}=-\omega_{j} E_{ \pm} \quad(1 \leq j \leq 2 n+1) .
\end{aligned}
$$

Definition 6.2. The algebra $\mathcal{A}_{\Lambda}(n)$, when $\Lambda$ is a formal parameter, is defined in a similar way: it is the algebra with generators $\omega_{1}, \ldots, \omega_{2 n+1}, E_{ \pm}, \Lambda$ with $\Lambda$ central and same relations as $\mathcal{A}_{\lambda}(n)$ with $\lambda$ replaced by $\Lambda$. Note that $\mathcal{A}_{\Lambda}(0)=\mathcal{A}_{\Lambda}$. 


\subsection{Construction using Ore extensions}

Definition 6.3. Suppose that $R$ is an algebra, $\sigma$ an automorphism of $R$ and $\delta$ a $\sigma$-derivation of $R$, that is, a linear map $\delta: R \rightarrow R$ such that

$$
\delta(r s)=\delta(r) s+\sigma(r) \delta(s)
$$

for all $r, s \in R$. Then the Ore extension $R[t]$ is the free left $R$-module on the set $\left\{t^{n} \mid n \geq 0\right\}$, with multiplication defined by

$$
t r=\sigma(r) t+\delta(r)
$$

Let $\mathcal{C}=\mathcal{C}(2 n+1)$ be the Clifford algebra in $2 n+1$ generators, $\omega_{1}, \ldots, \omega_{2 n+1}$. Consider the polynomial ring $\mathcal{C}[\Lambda]$ where $\Lambda$ commutes with all elements of $\mathcal{C}$. Elements of $\mathcal{C}[\Lambda]$ are denoted by $C(\Lambda)$.

Let $\tau$ be the automorphism of $\mathcal{C}[\Lambda]$ defined by

$$
\tau\left(\omega_{r}\right)=-\omega_{r}, \quad \forall r \quad \text { and } \quad \tau(\Lambda)=\Lambda .
$$

The free $\mathcal{C}[\Lambda]$-module $\mathcal{C}[\Lambda]\left[E_{+}\right]$with basis $\left\{E_{+}^{n} \mid n \in \mathbb{N}\right\}$ gives us a a first Ore extension with

$$
E_{+} C(\Lambda)=\tau(C(\Lambda)) E_{+}, \quad \forall C(\Lambda) \in \mathcal{C}[\Lambda]
$$

The following lemma is easy:

Lemma 6.4. There exists an automorphism $\sigma$ of the Ore extension $\mathcal{C}[\Lambda]\left[E_{+}\right]$satisfying:

$$
\sigma\left(E_{+}\right)=E_{+}, \quad \sigma\left(\omega_{r}\right)=-\omega_{r}, \quad \forall r \quad \text { and } \quad \sigma(\Lambda)=\Lambda .
$$

Let $\theta$ be the element $i^{n} \omega_{1} \cdots \omega_{2 n+1} \Lambda$ in $\mathcal{C}[\Lambda]$. So $\theta$ commutes with $\Lambda$ and $\omega_{r}, \forall r$ and anticommutes with $E_{+}$.

Let $\Delta$ be the operator of $\mathcal{C}\left[E_{+}\right]$defined by

$$
\Delta(f)=\frac{f\left(E_{+}\right)-f\left(-E_{+}\right)}{2 E_{+}}, \quad \forall f \in \mathcal{C}\left[E_{+}\right]
$$

and $D$ be the operator of $\mathcal{C}[\Lambda]\left[E_{+}\right]$defined by

$$
D\left(f\left(E_{+}\right) C(\Lambda)\right)=\left(\frac{1}{4} \frac{d f}{d E_{+}}-\Delta(f) \theta\right) C(\Lambda), \quad \forall f \in \mathcal{C}\left[E_{+}\right], \quad C(\Lambda) \in \mathcal{C}[\Lambda] .
$$

Lemma 6.5. One has $D(A B)=\sigma(A) D(B)+D(A) B$ for all $A, B \in \mathcal{C}[\Lambda]\left[E_{+}\right]$.

Proof. This is a straightforward verification.

From Lemmas 6.4 and 6.5, we can now construct a second Ore extension $\mathcal{C}[\Lambda]\left[E_{+}\right]\left[E_{-}\right]$satisfying

$$
E_{-} A=\sigma(A) E_{-}+D(A), \quad \forall A \in \mathcal{C}[\Lambda]\left[E_{+}\right] .
$$

It follows that:

$$
\begin{aligned}
& {\left[E_{+}, E_{-}\right]_{\mathcal{L}}=-\frac{1}{4}+\theta,} \\
& \omega_{k} \omega_{j}+\omega_{j} \omega_{k}=2 \delta_{j k} \quad(1 \leq j, k \leq 2 n+1), \\
& E_{ \pm} \omega_{j}=-\omega_{j} E_{ \pm} \quad(1 \leq j \leq 2 n+1) .
\end{aligned}
$$




\section{Proposition 6.6.}

1. The Ore extension $\mathcal{C}[\Lambda]\left[E_{+}\right]\left[E_{-}\right]$and $\mathcal{A}_{\Lambda}(n)$ are isomorphic algebras.

2. A basis of $\mathcal{A}_{\Lambda}(n)$ is given by:

$$
\left\{\omega^{I} E_{+}^{\alpha} E_{-}^{\beta} \Lambda^{r} \mid I \in\{0,1\}^{2 n+1}, \alpha, \beta, r \in \mathbb{N}\right\},
$$

where $\omega^{I}=\omega_{1}^{i_{1}} \cdots \omega_{2 n+1}^{i_{2 n+1}}$ for all $I=\left(i_{1}, \ldots, i_{2 n+1}\right) \in\{0,1\}^{2 n+1}$.

If $\Lambda$ is replaced by a small $\lambda(\lambda \in \mathbb{C})$ in the definition of $\mathcal{A}_{\Lambda}(n)$, the same procedure works to construct an Ore extension of $\mathcal{C}\left[E_{+}\right]\left[E_{-}\right]$, isomorphic to $\mathcal{A}_{\lambda}(n)$. So

Proposition 6.7. A basis of $\mathcal{A}_{\lambda}(n)$ is given by:

$$
\left\{\omega^{I} E_{+}^{\alpha} E_{-}^{\beta} \mid I \in\{0,1\}^{2 n+1}, \alpha, \beta \in \mathbb{N}\right\} .
$$

The algebra $\mathcal{A}_{\lambda}(n)$ is the quotient $\mathcal{A}_{\Lambda}(n) / I_{\lambda}$ where $I_{\lambda}$ is the ideal $\mathcal{A}_{\Lambda}(n)(\Lambda-\lambda)$. As a particular case, setting $p=2 E_{-}$and $q=2 E_{+}$, we obtain:

$$
\mathcal{A}_{0}(n) \simeq \mathcal{C}(2 n+1,2) \simeq \mathcal{A}_{\Lambda}(n) / \Lambda \mathcal{A}_{\Lambda}(n)
$$

Since $\mathcal{A}_{\Lambda}(n)=\mathcal{A}_{0}(n)[\Lambda]$ as vector spaces, we obtain:

Proposition 6.8. The algebra $\mathcal{A}_{\Lambda}(n)$ is a non-trivial polynomial deformation of the CliffordWeyl algebra $\mathcal{A}_{0}(n)=\mathcal{C}(2 n+1,2)$.

Proof. We just have to show that the deformation is non-trivial, but that results from the fact that the deformation cocycle is non-trivial by Corollary 5.3.

Remark 6.9. From Corollary 5.3 and Lemma B.2, this polynomial deformation $\mathcal{A}_{\Lambda}(n)$ is a universal deformation formula of $\mathcal{C}(2 n+1,2)$.

Corollary 6.10. The center of $\mathcal{A}_{\Lambda}(n)$ is $\mathbb{C}[\Lambda]$. Moreover, $\mathcal{A}_{\Lambda}(n)$ and $\mathcal{A}_{\lambda}(n)$ are Noetherian algebras.

Proof. We have $\mathcal{A}_{0}(n) \simeq \mathcal{C}(2 n+1,2)$ with center $\mathbb{C}$ (Corollary 3.3). Let $\widetilde{a}$ be a central element of $\mathcal{A}_{\Lambda}(n)$. By Proposition 6.8 , we can write $\widetilde{a}=a_{0}+\Lambda \widetilde{b}$ with $a_{0} \in \mathcal{A}_{0}(n)$ and $\widetilde{b} \in \mathcal{A}_{\Lambda}(n)$. Therefore in $\mathcal{A}_{\Lambda}(n)$ :

$$
x a_{0}+\Lambda x \widetilde{b}=a_{0} x+\Lambda \widetilde{b} x, \quad \forall x \in \mathcal{A}_{0}(n) .
$$

But $x a_{0}=x \times a_{0}+\Lambda \widetilde{c}$ and $a_{0} x=a_{0} \times x+\Lambda \widetilde{d}$ where $\times$ denotes the product of $\mathcal{A}_{0}(n)$. So $a_{0}$ is central in $\mathcal{A}_{0}(n)$, henceforth $a_{0} \in \mathbb{C}$. It follows $\widetilde{b}$ is central in $\mathcal{A}_{\Lambda}(n)$ and repeating the same argument, we obtain $\widetilde{a} \in \mathbb{C}[\Lambda]$. Finally, $\mathcal{A}_{\Lambda}(n)$ and $\mathcal{A}_{\lambda}(n)$ are Noetherian since they are constructed by Ore extensions of Noetherian algebras [15].

In the sequel, we denote $\mathcal{A}_{\lambda}$ the algebra $\mathcal{A}_{\lambda}(0)$. The periodicity of Clifford algebras can be extended to the algebras $\mathcal{A}_{\Lambda}(n)$ and $\mathcal{A}_{\lambda}(n)$ :

Lemma 6.11 (Periodicity Lemma 2). One has

$$
\mathcal{A}_{\Lambda}(n) \simeq \mathcal{C}(2 n) \otimes \mathcal{A}_{\Lambda} \simeq \mathcal{M}_{2^{n}}\left(\mathcal{A}_{\Lambda}\right) \quad \text { and } \quad \mathcal{A}_{\lambda}(n) \simeq \mathcal{C}(2 n) \otimes \mathcal{A}_{\lambda} \simeq \mathcal{M}_{2^{n}}\left(\mathcal{A}_{\lambda}\right)
$$


Proof. We denote by $P$ and $E_{ \pm}$the generators of $\mathcal{A}_{\Lambda}(0)$ satisfying $P E_{ \pm}=-E_{ \pm} P, P^{2}=1$ and $\left[E_{+}, E_{-}\right]_{\mathcal{L}}=-\frac{1}{4}+\Lambda P$. Let $\omega_{1}, \ldots, \omega_{2 n}$ be the generators of $\mathcal{C}(2 n)$.

We define $\omega_{1}^{\prime}, \ldots, \omega_{2 n+1}^{\prime}$ and $E_{ \pm}^{\prime}$ elements of $\mathcal{C}(2 n) \otimes \mathcal{A}_{\Lambda}(0)$ by:

$$
\omega_{i}^{\prime}=\omega_{i} \otimes P \quad(1 \leq i \leq 2 n), \quad \omega_{2 n+1}^{\prime}=i^{n} \omega_{1} \cdots \omega_{2 n} \otimes P, \quad E_{ \pm}^{\prime}=1 \otimes E_{ \pm} .
$$

Using $\left(\omega_{1} \cdots \omega_{2 n}\right)^{2}=(-1)^{n}$, we check that $\omega_{1}^{\prime}, \ldots, \omega_{2 n+1}^{\prime}$ verify the defining relations of $\mathcal{C}(2 n+1)$ and anti-commute with $E_{ \pm}^{\prime}$. The relation $\left[E_{+}^{\prime}, E_{-}^{\prime}\right]_{\mathcal{L}}=-\frac{1}{4}+i^{n} \Lambda \omega_{1}^{\prime} \cdots \omega_{2 n+1}^{\prime}$ results from $1 \otimes P=i^{n} \omega_{1}^{\prime} \cdots \omega_{2 n+1}^{\prime}$.

Finally, this last equality and the fact that $\omega_{i}=\omega_{i}^{\prime}(1 \otimes P)$ imply that $\omega_{i}^{\prime}(1 \leq i \leq 2 n+1)$ and $E_{ \pm}^{\prime}$ generate the algebra $\mathcal{C}(2 n) \otimes \mathcal{A}_{\Lambda}(n)$.

On the other hand, if $\omega_{1}, \ldots, \omega_{2 n+1}, E_{ \pm}$are the generators of $\mathcal{A}_{\Lambda}(n)$, we define $\omega_{1}^{\prime}, \ldots, \omega_{2 n}^{\prime}$, $E_{ \pm}^{\prime}$ and $P^{\prime}$ by:

$$
P^{\prime}=i^{n} \omega_{1} \cdots \omega_{2 n+1}, \quad \omega_{i}^{\prime}=\omega_{i} P^{\prime} \quad(1 \leq i \leq 2 n), \quad E_{ \pm}^{\prime}=E_{ \pm} .
$$

Since $P^{\prime}$ commutes with $\omega_{i}$, it commutes with $\omega_{i}^{\prime}$. Since $E_{ \pm}^{\prime}$ anti-commute with $\omega_{i}$, they anticommute with $P^{\prime}$ and commute with $\omega_{i}^{\prime}$. The equality $P^{\prime 2}=1$ follows from $\left(\omega_{1} \cdots \omega_{2 n+1}\right)^{2}=$ $(-1)^{n}$ and we conclude $\omega_{i}^{\prime 2}=1$. Moreover $\omega_{i}^{\prime}$ anti-commutes with $\omega_{j}^{\prime}$ for $i \neq j$ and $\left[E_{+}^{\prime}, E_{-}^{\prime}\right]_{\mathcal{L}}=$ $-\frac{1}{4}+i^{n} \Lambda \omega_{1} \cdots \omega_{2 n+1}=-\frac{1}{4}+\Lambda P^{\prime}$.

All defining relations of $\mathcal{C}(2 n) \otimes \mathcal{A}_{\Lambda}(0)$ are satisfied. Moreover $\omega_{i}=\omega_{i}^{\prime} P^{\prime}$ and $i^{n}\left(\omega_{1}^{\prime} \cdots \omega_{2 n+1}^{\prime}\right) P^{\prime}$ $=i^{2 n}\left(\omega_{1} \cdots \omega_{2 n}\right)^{2} P^{\prime 2 n} \omega_{2 n+1}=\omega_{2 n+1}$. So we conclude that $\omega_{i}^{\prime}, E_{ \pm}$and $P^{\prime}$ generate $\mathcal{A}_{\Lambda}(n)$.

This ends the proof that $\mathcal{A}_{\Lambda}(n) \simeq \mathcal{C}(2 n) \otimes \mathcal{A}_{\Lambda}$. Since $\mathcal{C}(2 n) \simeq \mathcal{M}_{2^{n}}(\mathbb{C})$, then $\mathcal{A}_{\Lambda}(n) \simeq$ $\mathcal{M}_{2^{n}}\left(\mathcal{A}_{\Lambda}\right)$.

A similar reasoning works for $\mathcal{A}_{\lambda}(n)$.

Remark 6.12. The first isomorphism in Lemma 6.11 is not a surprise: if $A$ is an algebra, all deformations of $\mathcal{M}_{k}(A)$ are of type $\mathcal{M}_{k}\left(A_{\Lambda}\right)$ where $A_{\Lambda}$ is a deformation of $A$ (see Appendix $\mathrm{C}$ ). Here, $\mathcal{C}(2 n+1,2) \simeq \mathcal{M}_{2^{n}}(\mathcal{C}(1,2))$ and $\mathcal{A}_{\Lambda}$ is a deformation of $\mathcal{C}(1,2)$.

\section{Algebras $\mathcal{A}_{\Lambda}(n)$ and their representations}

Let $\left\{E_{+}, E_{-}, Y, F, G\right\}$ be the usual generators of the Lie superalgebra osp $(1,2)$ : one has $\mathfrak{o s p}(1,2)_{\overline{0}}$ $=\operatorname{span}\{Y, F, G\}, \mathfrak{o s p}(1,2)_{\overline{1}}=\operatorname{span}\left\{E_{+}, E_{-}\right\}$and the commutation relations

$$
\begin{aligned}
& {\left[Y, E_{ \pm}\right]= \pm \frac{1}{2} E_{ \pm}, \quad[Y, F]=F, \quad[Y, G]=-G, \quad[F, G]=2 Y,} \\
& {\left[F, E_{+}\right]=\left[G, E_{-}\right]=0, \quad\left[F, E_{-}\right]=-E_{+}, \quad\left[G, E_{+}\right]=-E_{-},} \\
& {\left[E_{+}, E_{+}\right]=F, \quad\left[E_{-}, E_{-}\right]=-G, \quad\left[E_{+}, E_{-}\right]=Y,}
\end{aligned}
$$

where $[\cdot, \cdot]$ denotes the super bracket.

Let $\mathcal{U}:=\mathcal{U}(\mathfrak{o s p}(1,2))$ be the enveloping algebra of $\mathfrak{o s p}(1,2)$. Denote by $\theta \in \mathcal{U}$ the ghost:

$$
\theta:=\frac{1}{4}+\left[E_{+}, E_{-}\right]_{\mathcal{L}},
$$

where $[\cdot, \cdot]_{\mathcal{L}}$ denotes the Lie bracket.

Lemma $7.1([20,2,3])$. The relation $\theta E_{ \pm}=-E_{ \pm} \theta$ holds in $\mathcal{U}$.

Proof. We have $\theta=\frac{1}{4}+E_{+} E_{-}-E_{-} E_{+}$, hence

$$
E_{+} \theta=\frac{1}{4} E_{+}+E_{+}^{2} E_{-}-E_{+} E_{-} E_{+}, \quad \theta E_{+}=\frac{1}{4} E_{+}+E_{+} E_{-} E_{+}-E_{-} E_{+}^{2} .
$$

Therefore $E_{+} \theta+\theta E_{+}=\frac{1}{2} E_{+}-\left[Y, E_{+}\right]=0$. Similarly, we can prove that $E_{-} \theta=-E_{-} \theta$. 
Let us now consider the $\mathbb{C}$-algebra $\mathcal{U}_{\vartheta}$ defined by:

$$
\mathcal{U}_{\vartheta}:=\left\langle\mathcal{E}_{+}, \mathcal{E}_{-}, \vartheta \mid\left[\mathcal{E}_{+}, \mathcal{E}_{-}\right]_{\mathcal{L}}=-\frac{1}{4}+\vartheta, \mathcal{E}_{ \pm} \vartheta=-\vartheta \mathcal{E}_{ \pm}\right\rangle .
$$

By Lemma 7.1, the enveloping algebra $\mathcal{U}$ is a quotient of $\mathcal{U}_{\vartheta}$.

Proposition 7.2 ([13]). There exists an algebra isomorphism between $\mathcal{U}$ and $\mathcal{U}_{\vartheta}$.

Proof. Consider the subspace $V=V_{\overline{0}} \oplus V_{\overline{1}}$ of $\mathcal{U}_{\vartheta}$, with $V_{\overline{0}}=\{0\}$ and $V_{\overline{1}}=\operatorname{span}\left\{\mathcal{E}_{+}, \mathcal{E}_{-}\right\}$. Define a supersymmetric bilinear form $(\cdot, \cdot)$ on $V$ (hence symplectic on $V_{\overline{1}}$ ) by:

$$
\left(\mathcal{E}_{+}, \mathcal{E}_{-}\right)=-\frac{1}{4}, \quad\left(\mathcal{E}_{+}, \mathcal{E}_{+}\right)=\left(\mathcal{E}_{-}, \mathcal{E}_{-}\right)=0 .
$$

The algebra $\mathcal{U}_{\vartheta}$ is $\mathbb{Z}_{2}$-graded by the $\mathbb{Z}_{2}$-gradation of $V$. Starting from $\left[\mathcal{E}_{+}, \mathcal{E}_{-}\right]=2 \mathcal{E}_{+} \mathcal{E}_{-}+\frac{1}{4}-\theta$ with $\mathcal{E}_{ \pm} \theta=-\theta \mathcal{E}_{ \pm}$, we have:

$$
\left[\left[\varepsilon_{+}, \mathcal{E}_{-}\right], \varepsilon_{ \pm}\right]= \pm \frac{1}{2} \varepsilon_{ \pm}
$$

Using the Jacobi identity, we get $\left[\left[\mathcal{E}_{+}, \mathcal{E}_{+}\right], \mathcal{E}_{-}\right]=-2\left[\left[\mathcal{E}_{+}, \mathcal{E}_{-}\right], \mathcal{E}_{+}\right]=-\mathcal{E}_{+}$and $\left[\left[\mathcal{E}_{+}, \mathcal{E}_{+}\right], \mathcal{E}_{+}\right]=0$. In the same way, $\left[\left[\mathcal{E}_{-}, \mathcal{E}_{-}\right], \mathcal{E}_{+}\right]=\mathcal{E}_{-}$and $\left[\left[\mathcal{E}_{-}, \mathcal{E}_{-}\right], \mathcal{E}_{-}\right]=0$. We conclude that

$$
[[X, Y], Z]=2((Y, Z) X+(X, Z) Y), \quad \forall X, Y \in V_{\overline{1}} .
$$

By Corollary 4.2, we deduce a surjective algebra homomorphism from $\mathcal{U}$ to $\mathcal{U}_{\vartheta}$ and using Lemma 7.1, we finish the proof.

\section{Proposition 7.3.}

1. $\mathcal{A}_{\Lambda}(n) \simeq \mathcal{C}(2 n+1) \underset{\mathbb{Z}_{2}}{\otimes} \mathcal{U}$.

2. $\mathcal{A}_{\Lambda}(n) \simeq \mathcal{C}(2 n) \otimes\left(\mathfrak{S}_{2} \ltimes \mathcal{U}\right) \simeq \mathcal{M}_{2^{n}}\left(\mathfrak{S}_{2} \ltimes \mathcal{U}\right)$.

Proof. 1. Let $\omega_{1}, \ldots, \omega_{2 n+1}$ be the generators of $\mathcal{C}(2 n+1)$. Here $\mathcal{C}(2 n+1)$ is $\mathbb{Z}_{2}$-graded by $\operatorname{deg}_{\mathbb{Z}_{2}}\left(\omega_{i}\right)=1, \forall i$. Define $\Lambda \in \mathcal{C}(2 n+1) \underset{\mathbb{Z}_{2}}{\otimes \mathcal{U}}$ by

$$
\Lambda=i^{n} \omega_{1} \cdots \omega_{2 n+1} \theta .
$$

We see immediately that $\Lambda$ is a central element and that $\omega_{1}, \ldots, \omega_{2 n+1}, E_{ \pm}$and $\Lambda$ satisfy the defining relations of $\mathcal{A}_{\Lambda}(n)$. Moreover, they generate $\mathcal{C}(2 n+1) \underset{\mathbb{Z}_{2}}{\otimes} \mathcal{U}$ since $\theta=i^{n} \omega_{1} \cdots \omega_{2 n+1} \Lambda$. Then there exists a surjective algebra homomorphism from $\mathcal{A}_{\Lambda}(n)$ to $\mathcal{C}(2 n+1) \otimes \mathcal{U}$.

To define the inverse map, we introduce an element $\theta \in \mathcal{A}_{\Lambda}(n)$ by

$$
\theta=i^{n} \omega_{1} \cdots \omega_{2 n+1} \Lambda
$$

To finish the proof, we notice that elements $E_{+}, E_{-}$and $\theta$ verify the defining relations of $\mathcal{U}_{\vartheta} \simeq \mathcal{U}$, hence $\omega_{1}, \ldots, \omega_{2 n+1}, E_{ \pm}$and $\theta$ satisfy the defining relations of $\mathcal{C}(2 n+1) \underset{\mathbb{Z}_{2}}{\otimes \mathcal{U}}$ and they generate $\mathcal{A}_{\Lambda}(n)$.

2. The parity of $\mathcal{U}$ is used to define the smash product $\mathfrak{S}_{2} \ltimes \mathcal{U}=\mathcal{C}(1) \underset{\mathbb{Z}_{2}}{ } \mathcal{U}$. To prove (2), apply Lemma 6.11 and (1).

Remark 7.4. The algebra $\mathcal{A}_{\Lambda}$ is a deformation of $\mathcal{C}(1,2)=\mathfrak{S}_{2} \ltimes \mathrm{W}_{2}$. Besides $\mathcal{A}_{\Lambda}=\mathfrak{S}_{2} \ltimes \mathcal{U}$. So here is a particular case where a deformation of a smash product remains a smash product. Moreover, representations of $\mathcal{A}_{\Lambda}$ are merely graded representations of $\mathcal{U}$. 
Definition 7.5. An algebra $A$ is a FCR algebra if:

1. Every finite-dimensional representation of $A$ is completely reducible.

2. The intersection of all kernels of finite-dimensional representations is $\{0\}$.

If $A$ is an algebra and $V$ is an $A$-module, there is a corresponding structure of $\mathcal{M}_{n}(A)$-module on $\mathcal{V}=V^{n}$, since $n \times n$-matrices with coefficients in $A$ act on $n$-column vectors with coefficients in $V$. The following well-known Lemma shows that all $\mathcal{M}_{n}(A)$-modules are of this type:

Lemma 7.6. Given an $\mathcal{M}_{n}(A)$-module $\mathcal{V}$, there exists a corresponding A-module $V$ such that $\mathcal{V} \simeq V^{n}$, and $\operatorname{ker}(\mathcal{V})=\mathcal{M}_{n}(\operatorname{ker}(V))$.

Proof. Let $E_{i j}$ be the elementary matrices in $\mathcal{M}_{n}$. They satisfy $\operatorname{Id}=\sum_{i=1}^{n} E_{i i}$ and $E_{i i} E_{j j}=$ $\delta_{i j} E_{i i}$. So $\mathcal{V}=\oplus_{i=1}^{n} W_{i}$ where $W_{i}=E_{i i} \cdot \mathcal{V}$. Since $A$ and $\mathcal{M}_{n}$ commute, $W_{i}$ are $A$-modules. Since $E_{i j} \cdot W_{j}=E_{i i} \cdot\left(E_{i j} \cdot W_{j}\right), E_{i j}$ maps $W_{j}$ into $W_{i}$ and $E_{i j} W_{k}=\{0\}$ if $k \neq j$. Using $E_{j i} E_{i j}=E_{j j}$, it results that $W_{i}$ and $W_{j}$ are isomorphic $A$-modules, for all $i, j$. Let $V$ be any of the $A$-modules $W_{i}$, it is easy to verify that $\mathcal{V} \simeq V^{n}$ as $\mathcal{M}_{n}(A)$-modules. For the second claim, use the following easy statement: if $J$ is a two-sided ideal of $\mathcal{M}_{n}(A)$, there exists a two-sided ideal $I$ of $A$ such that $J=\mathcal{M}_{n}(I)$.

Remark 7.7. The above correspondence between $A$-modules and $\mathcal{M}_{n}(A)$-modules respects isomorphisms, irreducibility and direct sum decompositions.

Corollary 7.8. If $A$ is an FCR algebra, then $\mathcal{M}_{n}(A)$ is also a FCR algebra.

Proof. Apply Lemma 7.6 and the remark above.

\section{Corollary 7.9.}

1. The algebra $\mathcal{A}_{\Lambda}(n)$ is a FCR algebra.

2. All finite-dimensional representations of $\mathcal{A}_{\lambda}(n)$ are completely reducible.

Proof. 1. By Periodicity Lemma $2, \mathcal{A}_{\Lambda}(n) \simeq \mathcal{M}_{2^{n}}\left(\mathcal{A}_{\Lambda}\right)$ and by Proposition $7.3, \mathcal{A}_{\Lambda} \simeq \mathfrak{S}_{2} \ltimes \mathcal{U}$. Representations of $\mathfrak{S}_{2} \ltimes \mathcal{U}$ are simply $\mathbb{Z}_{2}$-graded representations of $\mathcal{U}$ and by [6], finite-dimensional ones are completely reducible. Moreover by [5], the intersection of all kernels of finitedimensional representations of $\mathfrak{S}_{2} \ltimes \mathcal{U}$ is $\{0\}$. So $\mathfrak{S}_{2} \ltimes \mathcal{U}$ is FCR, then apply Corollary 7.8 .

2. Use $\mathcal{A}_{\lambda}(n) \simeq \mathcal{A}_{\Lambda}(n) /(\Lambda-\lambda) \mathcal{A}_{\Lambda}(n)$.

Remark 7.10. By Lemma 7.6, the representation theory of $\mathcal{A}_{\Lambda}(n) \simeq \mathcal{M}_{2^{n}}\left(\mathcal{A}_{\Lambda}\right)$ is reduced to the representation theory of $\mathcal{A}_{\Lambda} \simeq \mathfrak{S}_{2} \ltimes \mathcal{U}$ and therefore to the $\mathbb{Z}_{2}$-graded representation theory of $\mathcal{U}$.

Example 7.11. To study finite-dimensional representations of $\mathcal{A}_{\Lambda}(n)$, it is enough to study irreducible ones by Corollary 7.9. Irreducible representations of $\mathfrak{o s p}(1,2)$ are well-known, they are all $\mathbb{Z}_{2}$-graded: given $h \in \frac{1}{2} \mathbb{N}$, there exists an irreducible representation on a $(4 h+1)$-dimensional space $V_{h}$ to which corresponds an irreducible representation of $\mathcal{A}_{\Lambda}(n)$ given by $2^{n} \times 2^{n}$-matrices with coefficients in $\mathfrak{S}_{2} \ltimes \mathcal{U}$ acting on $\mathcal{V}_{h}=V_{h}^{2^{n}}$, therefore of dimension $2^{n}(4 h+1)$. Alternatively, since $\mathcal{A}_{\Lambda}(n) \simeq \mathcal{C}(2 n) \otimes \mathcal{A}_{\Lambda}$, this representation is the natural action of $\mathcal{C}(2 n) \otimes \mathcal{A}_{\Lambda}$ on $\mathcal{V}_{h}=\Phi_{n} \otimes V_{h}$ where $\Phi_{n}$ is the spin representation of $\mathcal{C}(2 n)$ (see Section 1). This describes all finite-dimensional representations of $\mathcal{A}_{\Lambda}(n)$. 
Example 7.12. We will now construct examples of simple $\mathcal{A}_{\lambda}$-modules from the metaplectic representation of $\mathrm{W}_{2}$. Let $V=\mathbb{C}[z]$ as in [13]. We define the operator $\Delta$ of $V$ :

$$
\Delta(h)=\frac{1}{z}(h(z)-h(-z)), \quad \forall h \in V .
$$

Denote by $P$ the parity operator of $V$. Define operators $\rho_{\lambda}^{ \pm}$by:

$$
\rho_{\lambda}^{+}=\frac{1}{2} \frac{d}{d z}-\lambda \Delta, \quad \rho_{\lambda}^{-}=-\frac{1}{2} z .
$$

Then $\left[\rho_{\lambda}^{+}, \rho_{\lambda}^{-}\right]_{\mathcal{L}}=-\frac{1}{4}+\lambda P, \rho_{\lambda}^{ \pm} P=-P \rho_{\lambda}^{ \pm}$and $P^{2}=1$. In this way, we obtain a representation $\rho_{\lambda}$ of $\mathcal{A}_{\lambda}$ in $V$ such that:

$$
\rho_{\lambda}\left(E_{ \pm}\right)=\rho_{\lambda}^{ \pm}, \quad \rho_{\lambda}(P)=P .
$$

We recover exactly the $\mathbb{Z}_{2}$-graded $\mathfrak{o s p}(1,2)$-Verma module $\mathcal{V}_{\lambda-\frac{1}{4}}$ of highest weight $\lambda-\frac{1}{4}$.

If $\lambda \neq h+\frac{1}{4}, 2 h \in \mathbb{N}$, then $\rho_{\lambda}\left(E_{+}\right)$does not vanish and the corresponding module is simple. If $\lambda=h+\frac{1}{4}, 2 h \in \mathbb{N}$, we have $\rho_{\lambda}\left(E_{+}\right)\left(z^{4 h+1}\right)=0$. Therefore $W_{h}=\operatorname{span}\left\{z^{\ell}, \ell \geq 4 h+1\right\}$ is a simple submodule of dominant weight $-\left(h+\frac{1}{2}\right)$, the quotient $V / W_{h}$ is the simple osp $(1,2)$-module of dimension $4 h+1$ and the module $\left(V, \rho_{h+\frac{1}{4}}\right)$ is a non-trivial extension of $W_{h}$ by $V / W_{h}$ (see [13] for more details).

Denote by $V_{\lambda}$ the $\mathcal{A}_{\lambda}$-module just built. Using $\mathcal{A}_{\lambda}(n)=\mathcal{M}_{2^{n}}\left(\mathcal{A}_{\lambda}\right)$, define a corresponding $\mathcal{A}_{\lambda}(n)$-module by setting $V_{\lambda}(n)=\Phi_{2 n} \otimes V_{\lambda}$ where $\Phi_{2 n}$ is the spin representation of $\mathcal{C}(2 n)$. When $\lambda \neq\left(h+\frac{1}{4}\right), 2 h \in \mathbb{N}$, we obtain a simple $\mathcal{A}_{\lambda}(n)$-module. When $\lambda=h+\frac{1}{4}, 2 h \in \mathbb{N}$, we obtain an indecomposable $\mathcal{A}_{\lambda}(n)$-module with a unique simple submodule and a unique simple quotient of dimension $2^{n}(4 h+1)$.

Since $\mathcal{A}_{\Lambda}(n) \simeq \mathcal{A}_{\Lambda}(n) /(\Lambda-\lambda) \mathcal{A}_{\Lambda}(n)$, these modules are $\mathcal{A}_{\Lambda}(n)$-modules.

Remark 7.13. When $A$ is a $\mathbb{Z}_{2}$-graded algebra, $\mathcal{M}_{n}(A)=\mathcal{M}_{n} \otimes A$ has a natural $\mathbb{Z}_{2}$-gradation induced by the gradation of $A$ and $\operatorname{deg}(M)=\overline{0}$ for all $M \in \mathcal{M}_{n}$. But algebras $\mathfrak{S}_{2} \ltimes \mathcal{M}_{n}(A)$ and $\mathcal{M}_{n}\left(\mathfrak{S}_{2} \ltimes A\right)$ have the same underlying vector space. It is easy to verify that they coincide as algebras. Using Proposition 7.3, Remark 3.4 and Lemma 7.6, it results that all representations of $\mathcal{A}_{\Lambda}(n)$ are graded and obtained from graded representations of $\mathcal{U}$.

\section{Algebras $\mathcal{A}_{\lambda}(n)$}

We keep the notation of last Section. Write $\mathfrak{g}=\mathfrak{o s p}(1,2)$ as $\mathfrak{g}=\mathfrak{g}_{\overline{0}} \oplus \mathfrak{g}_{\overline{1}}$ where $\mathfrak{g}_{\overline{0}}=\operatorname{span}\{Y, F, G\}$ and $\mathfrak{g}_{\overline{1}}=\operatorname{span}\left\{E_{+}, E_{-}\right\}, \mathcal{U}=\mathcal{U}(\mathfrak{g})$ its enveloping algebra and $\theta=\frac{1}{4}+\left[E_{+}, E_{-}\right]_{\mathcal{L}}$ the ghost. We have $\mathcal{Z}(\mathfrak{g})=\mathbb{C}[C]$ where $\mathcal{Z}(\mathfrak{g})$ denotes the center of $\mathcal{U}, C=\theta^{2}-\frac{1}{16}$ and $\mathcal{Z}\left(\mathfrak{g}_{\overline{0}}\right)=\mathbb{C}[Q]$ where $\mathcal{Z}\left(\mathfrak{g}_{\overline{0}}\right)$ denotes the center of $\mathcal{U}\left(\mathfrak{g}_{\overline{0}}\right), Q=\left(\theta-\frac{1}{4}\right)\left(\theta+\frac{3}{4}\right)[20,2]$. For $c \in \mathbb{C}$, let $\mathcal{B}_{c}:=\mathcal{U} /(C-c) \mathcal{U}$.

Let us consider the $\mathbb{C}$-algebra $\mathcal{A}_{\lambda}:=\mathcal{A}_{\lambda}(0)$. Recall that:

$$
\mathcal{A}_{\lambda}=\left\langle E_{+}, E_{-}, P \mid P^{2}=1,\left[E_{+}, E_{-}\right]_{\mathcal{L}}=-\frac{1}{4}+\lambda P, E_{ \pm} P=-P E_{ \pm}\right\rangle .
$$

If $\lambda=0, \mathcal{A}_{0}$ is the Clifford-Weyl algebra $\mathcal{C}(1,2)=\mathfrak{S}_{2} \ltimes \mathrm{W}_{2}$. In general:

Proposition 8.1. One has $\mathcal{A}_{\lambda} \simeq \mathcal{B}_{\lambda^{2}-\frac{1}{16}}$ whenever $\lambda \neq 0$.

Proof. For $u \in \mathcal{U}$, we denote by $\bar{u}$ its class in $\mathcal{B}_{\lambda^{2}-\frac{1}{16}}$. Therefore $\left[\overline{E_{+}}, \overline{E_{-}}\right]_{\mathcal{L}}=-\frac{1}{4}+\bar{\theta}$ and $\overline{E_{ \pm}} \bar{\theta}= \pm \bar{\theta} \overline{E_{ \pm}}$. Moreover, $\bar{C}=\lambda^{2}-\frac{1}{16}=\bar{\theta}^{2}-\frac{1}{16}$. Setting $P=\frac{1}{\lambda} \bar{\theta}$, one recovers exactly the defining relations of $\mathcal{A}_{\lambda}$ and a map from $\mathcal{A}_{\lambda}$ onto $\mathcal{B}_{\lambda^{2}-\frac{1}{16}}$.

For the inverse map, one can check that elements $E_{+}$and $E_{-}$in $\mathcal{A}_{\lambda}$ generate a superalgebra isomorphic to $\mathfrak{g}$, hence a homomorphism $\rho$ from $\mathcal{U}$ to $\mathcal{A}_{\lambda}$. We have $\rho(\theta)=\lambda P$, so $\rho$ is surjective. Since $\rho\left(C-\lambda^{2}+\frac{1}{16}\right)=0$, one can define the inverse map $\bar{\rho}$ from $\mathcal{B}_{\lambda^{2}-\frac{1}{16}}$ onto $\mathcal{A}_{\lambda}$. 
The structure of the algebra $\mathcal{A}_{\lambda}$ is deduced from the Proposition above and [20].

\section{Proposition 8.2.}

1. If $\lambda^{2} \neq\left(h+\frac{1}{4}\right)^{2}, 2 h \in \mathbb{N}$, then $\mathcal{A}_{\lambda}$ is a simple algebra.

2. If $\lambda^{2}=\left(h+\frac{1}{4}\right)^{2}, 2 h \in \mathbb{N}$, then $\mathcal{A}_{\lambda}$ is a primitive algebra. Moreover, there exists a unique non-zero two-sided ideal $I_{\lambda}$ in $\mathcal{A}_{\lambda}$ of codimension $(4 h+1)^{2}$, with $I_{\lambda}=\operatorname{ker}\left(V_{h}\right)$ and $V_{h}$ is the simple osp $(1,2)$-module of dimension $4 h+1$.

Proof. It is proved in [20] that $\mathcal{B}_{c}, c \neq 0$ has the following properties:

- if $c \neq h \frac{(2 h+1)}{2}, 2 h \in \mathbb{N}$, then $\mathcal{B}_{c}$ is $\mathbb{Z}_{2}$-simple;

- if $c=h \frac{(2 h+1)}{2}, 2 h \in \mathbb{N}$, then $\mathcal{B}_{c}$ is primitive. Moreover, there exists a unique non-zero $\mathbb{Z}_{2}$-graded two-sided ideal with codimension $(4 h+1)^{2}$ that is the kernel of the simple $\mathfrak{o s p}(1,2)$-module of dimension $4 h+1$.

If $\lambda=0$, then $\mathcal{A}_{0} \simeq \mathcal{C}(1,2)$ is simple.

If $\lambda \neq 0$, then $\mathcal{A}_{\lambda} \simeq \mathcal{B}_{\lambda^{2}-\frac{1}{16}}$. It is enough to show that any two-sided ideal of $\mathcal{B}_{\lambda^{2}-\frac{1}{16}}$ is $\mathbb{Z}_{2}$-graded and then translate the results just above in term of $\lambda$. So, let $I$ be a two-sided ideal of $\mathcal{B}_{\lambda^{2}-\frac{1}{16}}$. We set $P=\frac{1}{\lambda} \bar{\theta}$. We have $P^{2}=1$ and $P b P=(-1)^{\operatorname{deg}_{\mathbb{Z}_{2}}(b)} b, \forall b \in \mathcal{B}_{\lambda^{2}-\frac{1}{16}}$. If $a=a_{\overline{0}}+a_{\overline{1}} \in I$, it follows $P a P=a_{\overline{0}}-a_{\overline{1}} \in I$, therefore $a_{\overline{0}}$ and $a_{\overline{1}} \in I$.

\section{Corollary 8.3.}

1. If $\lambda^{2} \neq\left(h+\frac{1}{4}\right)^{2}, 2 h \in \mathbb{N}$, then $\mathcal{A}_{\lambda}(n)$ is a simple algebra.

2. If $\lambda^{2}=\left(h+\frac{1}{4}\right)^{2}, 2 h \in \mathbb{N}$, then $\mathcal{A}_{\lambda}(n)$ is a primitive algebra. Moreover, there exists a unique non-zero two-sided ideal in $\mathcal{A}_{\lambda}(n)$ of codimension $2^{2 n}(4 h+1)^{2}$, that is the kernel of the irreducible representation of dimension $2^{n}(4 h+1)$.

Proof. By Lemma $6.11, \mathcal{A}_{\lambda}(n) \simeq \mathcal{M}_{2^{n}}\left(\mathcal{A}_{\lambda}\right)$, so two-sided ideals of $\mathcal{A}_{\lambda}(n)$ are all of type $\mathcal{M}_{2^{n}}(I)$, $I$ is a two-sided ideal of $\mathcal{A}_{\lambda}$. Then apply Proposition 8.2 and Example 7.12.

Remark 8.4. We have $\mathcal{A}_{\Lambda}(n) \simeq \mathcal{A}_{\Lambda}(n) /(\Lambda-\lambda) \mathcal{A}_{\Lambda}(n)$. Moreover, the center of $\mathcal{A}_{\Lambda}(n)$ is $\mathbb{C}[\Lambda]$, so Corollary 8.3 lists all primitive quotients of $\mathcal{A}_{\Lambda}(n)$.

The algebra $\mathcal{A}_{0}=\mathcal{C}(1,2)$ is a quotient of $\mathcal{U}(\mathfrak{o s p}(2,2))$ (see Proposition 4.1). More generally:

Proposition 8.5. The algebra $\mathcal{A}_{\Lambda}$ is a quotient of $\mathfrak{S}_{2} \ltimes \mathcal{U}(\mathfrak{o s p}(2,2))$. Moreover, the Casimir operator of $\mathfrak{o s p}(2,2)$ (see [2]) vanishes in this quotient.

Proof. Using the notation in Section 6, we consider in $\mathcal{A}_{\Lambda}$ :

$$
K=-\frac{1}{4} \omega_{1}+\Lambda, \quad H_{\overline{0}}=\mathbb{C} K, \quad H_{\overline{1}}=\operatorname{span}\left\{E_{+}, E_{-}\right\}, \quad H=H_{\overline{0}} \oplus H_{\overline{1}} .
$$

Define $(\cdot \mid \cdot)$ a supersymmetric bilinear form on $H$ by $(K \mid K)=\frac{1}{8}$ and $\left(E_{+} \mid E_{-}\right)=-\frac{1}{4}$. It is easy to check that relations (PS) hold in $H$, so by Corollary 4.2, the subalgebra of $\mathcal{A}_{\Lambda}$ generated by $H$ is a quotient of $\mathcal{U}(\mathfrak{o s p}(2,2))$. Now, the subalgebra of $\mathcal{A}_{\Lambda}$ generated by $H$ and $\omega_{1}$ is $\mathcal{A}_{\Lambda}$ itself, and it is clearly a quotient of $\mathfrak{S}_{2} \ltimes \mathcal{U}(\mathfrak{o s p}(2,2))$. The second claim results from a direct computation using the Casimir formula given in [2].

Corollary 8.6. Any graded $\mathfrak{o s p}(1,2)$-module can be extended to an osp $(2,2)$-module (with same underlying space). 
Proof. First, remark that given a graded algebra $A$, graded $A$-modules and $\mathfrak{S}_{2} \ltimes A$-modules are exactly the same notion.

Now, start with a graded $\mathfrak{o s p}(1,2)$-module with parity $P$. Recall that $\left[E_{+}, E_{-}\right]_{\mathcal{L}}=-\frac{1}{4}+\theta$ with $\theta E_{ \pm}=-E_{ \pm} \theta$. We define $\Lambda=\theta P$ and $\omega_{1}=P$ to obtain a graded $\mathcal{A}_{\Lambda}$-module. By Proposition 8.5, this module is a $\mathfrak{S}_{2} \ltimes \mathcal{U}(\mathfrak{o s p}(2,2))$-module, therefore a graded $\mathfrak{o s p}(2,2)$-module.

Remark 8.7. Let $\mathcal{C}$ be the Casimir element of $\mathcal{U}(\mathfrak{o s p}(2,2))$. It is proved in [2] that a simple $\mathfrak{o s p}(2,2)$-module is still simple as an $\mathfrak{o s p}(1,2)$-module if, and only if, $\mathcal{C}=0$.

\section{A Appendix}

For the convenience of the reader, we recall here some notions of Hochschild cohomology theory relating it to Gerstenhaber deformation theory of (associative) algebras [11, 12]. See [4, 27] for applications of deformation theory to quantization.

Let $A$ be an (associative) algebra. By Hochschild cohomology of $A$, we mean Hochschild cohomology with coefficients in $A$, defined as follows.

For $k>0, k$-cochains are $k$-linear maps from $A^{k}$ to $A$. When $k=0,0$-cochains are simply elements of $A$. We denote by $M^{k}(A)$ the space of $k$-cochains and by $M(A)=\oplus_{k \geq 0} M^{k}(A)$, the space of cochains. We define the Hochschild coboundary operator $d$ acting on $M(A)$ by:

- if $a \in A=M^{0}(A), d a=-\operatorname{ad}(a)$ where $\operatorname{ad}(a)(b):=[a, b]$, for all $a, b \in A$;

- if $\Omega \in M^{k}(A), k>0$ :

$$
\begin{aligned}
d \Omega\left(a_{1}, \ldots, a_{k+1}\right)= & a_{1} \Omega\left(a_{2}, \ldots, a_{k+1}\right)-\Omega\left(a_{1} a_{2}, a_{3}, \ldots, a_{k+1}\right) \\
& +\Omega\left(a_{1}, a_{2} a_{3}, \ldots, a_{k+1}\right)+\cdots+(-1)^{k+1} \Omega\left(a_{1}, \ldots, a_{k}\right) a_{k+1} .
\end{aligned}
$$

One has $d^{2}=0$. Let $B^{0}(A)=\{0\}, B^{k}(A)=d M^{k-1}(A), k>0$. Set $Z^{k}(A)=\operatorname{ker}\left(\left.d\right|_{M^{k}(A)}\right)$, $k \geq 0$ and $H^{k}(A)=Z^{k}(A) / B^{k}(A)$. Elements of $B^{k}(A)$ (resp. $Z^{k}(A)$ ) are $k$-coboundaries (resp. $k$-cocycles) and $H^{k}(A)$ is the $k^{\text {th }}$-space of Hochschild cohomology of $A$. Note that $H^{0}(A)$ is the center of $A$. Here are some examples of algebras $A$ such that $H^{k}(A)=\{0\}$ for all $k>0$ : semisimple algebras (e.g. algebras of finite groups, algebras of complex matrices, Clifford algebras), Weyl algebras, etc.

A deformation of $A$ with formal parameter $\Lambda$ is a $\mathbb{C}[[\Lambda]]$-algebra structure on $A[[\Lambda]]$ defined by:

$$
a \star b=a b+\sum_{n \geq 1} \Lambda^{n} \Omega_{n}(a, b), \quad \forall a, b \in A, \quad \Omega_{n} \in M^{2}(A), \quad \forall n .
$$

The associativity of $\star$ can be reinterpreted in terms of Hochschild cohomology: $\Omega_{1} \in Z^{2}(A)$ and when $\Omega_{1} \in B^{2}(A)$, it can be removed by an equivalence, i.e. an isomorphism of $\mathbb{C}[[\Lambda]]$ algebras. When $H^{2}(A)=\{0\}$, repeating the same argument, it results that any deformation is equivalent to the initial product, so $A$ is rigid. For instance, all algebras we just mentioned above are rigid. Second, the conditions on $\Omega_{n}, n \geq 2$ can be written in terms of 3-cohomology, and it results that if $H^{3}(A)=\{0\}$, then given any $\Omega_{1} \in Z^{2}(A)$, there exits a deformation with leading cocycle $\Omega_{1}$.

These two results are known as the rigidity and integrability theorems. 


\section{B Appendix}

The terminology and results presented in this Appendix are rather standard, but for the sake of completeness we include them here with proofs.

Let $A$ be an associative algebra with product $m_{0}$. Let $\mathrm{M}(A)=\sum_{k \geq 0} \mathrm{M}^{k}(A)$ be the space of multilinear maps from $A$ to $A$. The space $\mathrm{M}(A)$ is graded, $\mathrm{M}^{(k)}:=\mathrm{M}^{k+1}(A)$ and endowed with the Gerstenhaber bracket, it is a graded Lie algebra [22]. Let $d=-\operatorname{ad}\left(m_{0}\right)$. Since $d^{2}=0, d$ defines a complex on $\mathrm{M}(A)$, the Hochschild cohomology complex of $A$ (see [10]). Let $\mathrm{Z}^{2}(A)$ be the set of 2-cocycles, $B^{2}(A)$ the 2-coboundaries, and $H^{2}(A)$ chosen such that $\mathrm{Z}^{2}(A)=B^{2}(A) \oplus H^{2}(A)$.

Given two vector spaces $V$ and $W$, a formal map $F: V \rightarrow W$ is a power series $F=\sum_{k>0} F_{k}$ where $F_{k}$ is a homogeneous polynomial function of degree $k$ from $V$ to $W$. In the sequel, we will need essentially formal maps $F: H^{2}(A) \rightarrow \mathrm{M}(A)$ and we define a graded Lie algebra bracket coming from the one defined on $\mathrm{M}(A)$ by:

$$
\left[F, F^{\prime}\right]=\sum_{k \geq 0} \sum_{r+s=k}\left[F_{r}, F_{s}^{\prime}\right] \quad \text { for } \quad F=\sum_{k \geq 0} F_{k}, \quad F^{\prime}=\sum_{k \geq 0} F_{k}^{\prime}
$$

with $\left[F_{r}, F_{s}^{\prime}\right](h)=\left[F_{r}(h), F_{s}^{\prime}(h)\right], \forall h \in H^{2}(A)$.

Definition B.1. A universal deformation formula of $A$ is a formal map $F: \mathrm{Z}^{2}(A) \rightarrow \mathrm{M}^{2}(A)$ such that:

1) $F=m_{0}+\operatorname{Id}_{H^{2}(A)}+\sum_{k \geq 2} F_{k}$,

2) $[F, F]=0$.

If $F$ is a universal formula of deformation, $\lambda$ a formal parameter and $h \in H^{2}(A)$, then $m_{h}^{\lambda}:=F(\lambda h)=m_{0}+\lambda h+\sum_{k \geq 2} \lambda^{k} F_{k}$ is a deformation of $m_{0}$. More generally, if we have a formal curve in $H^{2}(A)[[\lambda]], \widetilde{h}=\sum_{n \geq 1} \lambda^{n} h_{n}$, then

$$
m_{\widetilde{h}}^{\lambda}:=F(\widetilde{h}(\lambda))=m_{0}+\lambda h_{1}+\sum_{k \geq 2} \lambda^{k} \sum_{\substack{i_{1}+\cdots+i_{n}=k \\ i_{1}, \ldots, i_{n} \geq 1,1 \leq n \leq k}} F_{n}\left(h_{i_{1}}, \ldots, h_{i_{n}}\right)
$$

is a deformation of $m_{0}$. The Lemma below is simply a translation of the classical criterion of integrability:

Lemma B.2. Let $\mathrm{D}^{2}(A)$ be a complementary subspace of $\mathrm{Z}^{2}(A)$ in $\mathrm{M}^{2}(A)$. If $H^{3}(A)=\{0\}$, then there exists a universal deformation formula

$$
F=m_{0}+\operatorname{Id}_{H^{2}(A)}+\sum_{k \geq 2} F_{k}, \quad \text { with } \quad F_{k} \in \mathrm{D}^{2}(A), \quad \forall k \geq 2 .
$$

Proof. Let $\sigma$ be a section of $d: \mathrm{M}^{2}(A) \rightarrow \mathrm{B}^{3}(A)$ such that $\sigma \circ d$ is the projection onto $\mathrm{D}^{2}(A)$ along $\mathrm{Z}^{2}(A)$. Step by step, we construct $F$ verifying $[F, F]=0, F=m_{0}+\operatorname{Id}_{H^{2}(A)}+\sum_{k \geq 2} F_{k}$ : first, we find $d\left(F_{2}\right)=\frac{1}{2}\left[\operatorname{Id}_{H^{2}(A)}, \operatorname{Id}_{H^{2}(A)}\right]$. Since $\left[\operatorname{Id}_{H^{2}(A)}, \operatorname{Id}_{H^{2}(A)}\right]$ is valued in $\mathrm{Z}^{3}(A)=\mathrm{B}^{3}(A)$, define a suitable $F_{2}=\frac{1}{2} \sigma \circ\left[\operatorname{Id}_{H^{2}(A)}, \operatorname{Id}_{H^{2}(A)}\right]$ (remark that $d \circ \sigma=\operatorname{Id}_{\mathrm{B}^{3}(A)}$ ). It is easy to see that the remaining $F_{k}$ can be constructed by the same procedure. 


\section{Lemma B.3.}

1. Let $m^{\lambda}$ be a deformation of $m_{0}$. Then, up to equivalence, $m^{\lambda}$ can be written as:

$$
m^{\lambda}=m_{0}+h(\lambda)+d(\lambda), \quad \text { with } \quad h(\lambda) \in \lambda H^{2}[[\lambda]], \quad d \in \lambda^{2} D^{2}[[\lambda]] .
$$

2. If $m^{\prime \lambda}$ is another deformation with

$$
m^{\prime \lambda}=m_{0}+h(\lambda)+d^{\prime}(\lambda), \quad \text { with } \quad d^{\prime}(\lambda) \in \lambda^{2} \mathrm{D}^{2}[[\lambda]]
$$

then $d^{\prime}(\lambda)=d(\lambda)$.

Proof. 1. Up to equivalence, we can assume that the leading cocycle of $m^{\lambda}$ is in $H^{2}(A)$, $m^{\lambda}=m_{0}+\lambda h_{1}+\lambda^{2} C_{2}+\cdots$.

We have $C_{2}=d_{2}+h_{2}+b_{2}, d_{2} \in \mathrm{D}^{2}(A), h_{2} \in H^{2}(A)$ and $b_{2} \in \mathrm{B}^{2}(A)$. We can assume that $b_{2}=0$, therefore $m^{\lambda}=m_{0}+\left(\lambda h_{1}+\lambda^{2} h_{2}\right)+\lambda^{2} d_{2}+\lambda^{3} C_{3}+\cdots$. Repeat the same argument to obtain the result.

2. Let $m^{\lambda}=m_{0}+\lambda h_{1}+\lambda^{2}\left(h_{2}+d_{2}\right)+\cdots, m_{\lambda}^{\prime}=m_{0}+\lambda h_{1}+\lambda^{2}\left(h_{2}+d_{2}^{\prime}\right)+\cdots$, then $d\left(h_{2}+d_{2}\right)=$ $\frac{1}{2}\left[h_{1}, h_{1}\right]=d\left(h_{2}+d_{2}^{\prime}\right)$, hence $d\left(d_{2}\right)=d\left(d_{2}^{\prime}\right)$ and that implies $d_{2}-d_{2}^{\prime} \in Z^{2}(A) \cap \mathrm{D}^{2}(A)=\{0\}$. Apply repeatedly the same reasoning to obtain $m_{\lambda}^{\prime}=m^{\lambda}$.

Proposition B.4. Assume that $H^{3}(A)=\{0\}$. Let $F$ be a universal deformation formula and $m^{\lambda}$ a deformation. Up to equivalence, there exists a formal curve $h(\lambda)$ in $H^{2}(A)[[\lambda]]$ such that $h(0)=0$ and $m^{\lambda}=F(h(\lambda))$. In other words, $F$ characterizes all deformations of $m_{0}$ up to equivalence and up to change of formal parameter.

Proof. The existence is given by the Lemma B.2. Up to equivalence, we can assume that $m^{\lambda}=m_{0}+h(\lambda)+d(\lambda)$ where $h(\lambda) \in \lambda H^{2}[[\lambda]]$ and $d(\lambda) \in \lambda^{2} H^{2}[[\lambda]]$ (Lemma B.3). But the deformation $m^{\prime \lambda}=F(h(\lambda))$ can be written as $m^{\prime \lambda}=m_{0}+h(\lambda)+d^{\prime}(\lambda)$ with $d^{\prime}(\lambda) \in \lambda^{2} H^{2}[[\lambda]]$. Henceforth $m^{\prime \lambda}=m^{\lambda}$ by Lemma B.3.

\section{Appendix}

Let $A$ be an algebra and $A_{\Lambda}$ be a deformation of $A$ with product $\star$. The underlying space of $A_{\Lambda}$ is $A[[\Lambda]]$, and it is easy to check that $\mathcal{M}_{n}(A[[\Lambda]])=\mathcal{M}_{n}(A)[[\Lambda]]$. Then $\mathcal{M}_{n}\left(A_{\Lambda}\right)$ is a deformation of $\mathcal{M}_{n}(A)$, the product is the natural one, defined by $(a M) \star\left(a^{\prime} M^{\prime}\right)=\left(a \star a^{\prime}\right) M M^{\prime}, \forall a, a^{\prime} \in A$, $M, M^{\prime} \in \mathcal{M}_{n}(A)$. Conversely:

Proposition C.1. Any deformation of $\mathcal{M}_{n}(A)$ is equivalent to a deformation $\mathcal{M}_{n}\left(A_{\Lambda}\right)$ with $A_{\Lambda}$ a deformation of $A$.

This result is known, but since we have not been able to find a reference, we give a short proof.

Proof. We refer to [10] for relative deformation theory with respect to a separable subalgebra. In the present case, the separable subalgebra of $\mathcal{M}_{n}(A)$ is $\mathcal{M}_{n}$, and any deformation is equivalent to a deformation with normalized $\mathcal{M}_{n}$-relative cochains [10], that is, cochains $\Omega:\left(\mathcal{M}_{n}(A)\right)^{2} \rightarrow$ $\mathcal{M}_{n}(A)$ that verify for all $M \in \mathcal{M}_{n}, a_{1}, a_{2} \in A$ :

$$
\begin{array}{ll}
\Omega\left(M a_{1}, a_{2}\right)=M \Omega\left(a_{1}, a_{2}\right), & \Omega\left(a_{1} M, a_{2}\right)=\Omega\left(a_{1}, M a_{2}\right), \\
\Omega\left(a_{1}, a_{2} M\right)=\Omega\left(a_{1}, a_{2}\right) M, & \text { and } \quad \Omega\left(x_{1}, x_{2}\right)=0 \quad \text { if one } \quad x_{i} \in \mathcal{M}_{n} .
\end{array}
$$


Since $\mathcal{M}_{n}$ and $A$ commute, such a cochain is completely determined by its restriction $\widetilde{\Omega}: A^{2} \rightarrow$ $\mathcal{M}_{n}(A)$ that verifies $M \widetilde{\Omega}\left(a_{1}, a_{2}\right)=\widetilde{\Omega}\left(a_{1}, a_{2}\right) M, \forall M \in \mathcal{M}_{n}, a_{1}, a_{2} \in A$, and is therefore $A$ valued. Summarizing, up to equivalence, we have a new product $\star$ that satisfies

$$
M_{1} \star M_{2}=M_{1} M_{2}, \quad M_{1} \star a=a \star M_{1}=a M_{1}, \quad\left(a_{1} M_{1}\right) \star\left(a_{2} M_{2}\right)=\left(a_{1} \star a_{2}\right) M_{1} M_{2}
$$

for all $M_{1}, M_{2} \in \mathcal{M}_{n}, a, a_{1}, a_{2} \in A$ and

$$
a_{1} \star a_{2}=a_{1} a_{2}+\sum_{n \geq 1} \Lambda^{n} C_{n}\left(a_{1}, a_{2}\right), \quad \forall a_{1}, a_{2} \in A
$$

with $C_{n}: A^{2} \rightarrow A$. So $\star$ defines a deformation $A_{\Lambda}$ of $A$.

Now, we will prove that our initial deformation $\star$ of $\mathcal{M}_{n}(A)$ is exactly the deformation $\mathcal{M}_{n}\left(A_{\Lambda}\right)$ : it is enough to show that $(a M) \star\left(a^{\prime} M^{\prime}\right)$ is the product of $(a M)$ and $\left(a^{\prime} M^{\prime}\right)$ in $\mathcal{M}_{n}\left(A_{\Lambda}\right)$, for all $a, a^{\prime} \in A, M, M^{\prime} \in \mathcal{M}_{n}$. But this is true since $a M \star a^{\prime} M^{\prime}=\left(a \star a^{\prime}\right) M^{\prime}$, that is exactly the product of $\mathcal{M}_{n}\left(A_{\Lambda}\right)$.

\section{References}

[1] Alev J., Farinati M.A., Lambre T., Solotar A.L., Homologie des invariants d'une algèbre de Weyl sous l'action d'un groupe fini, J. Algebra 232 (2000), 564-577.

[2] Arnal D., Benamor H., Pinczon G., The structure of $\mathfrak{s l}(2,1)$-supersymmetry: irreducible representations and primitive ideals, Pacific J. Math. 165 (1994), 17-49.

[3] Arnaudon D., Bauer L., Frappat L., On Casimir's ghost, Comm. Math. Phys. 187 (1997), 429-439, q-alg/9605021.

[4] Bayen F., Flato M., Fronsdal C., Lichnerowicz A., Sternheimer D., Deformation theory and quantization. I. Deformations of symplectic structures, Ann. Physics 111 (1978), 61-110.

Bayen F., Flato M., Fronsdal C., Lichnerowicz A., Sternheimer D., Deformation theory and quantization. II. Physical applications, Ann. Physics 111 (1978), 111-151.

[5] Behr E.J., Enveloping algebras of Lie superalgebras, Pacific J. Math. 130 (1987), 9-25.

[6] Djoković D.Ž., Hochschild G., Semisimplicity of 2-graded Lie algebras. II, Illinois J. Math. 20 (1976), 134143.

[7] Etingof P., Ginzburg V., Symplectic reflection algebras, Calogero-Moser space, and deformed HarishChandra homomorphism, Invent. Math. 147 (2002), 243-348, math.AG/0011114.

[8] Flato M., Fronsdal C., Parastatistics, highest weight $\mathfrak{o s p}(N, \infty)$ modules, singleton statistics and confinement, J. Geom. Phys. 6 (1989), 293-309.

[9] Fulton W., Harris J., Representation theory. A first course, Graduate Texts in Mathematics, Vol. 129, Springer-Verlag, New York, 1991.

[10] Gerstenhaber M., Schack D.S., Algebraic cohomology and deformation theory, in Deformation Theory of Algebras and Structures and Applications, Editors M. Hazewinkel and M. Gerstenhaber, NATO Advanced Science Institutes Series C: Mathematical and Physical Sciences, Vol. 247, Kluwer Academic Publishers Group, Dordrecht, 1988.

[11] Gerstenhaber M., On the deformation of rings and algebras, Ann. of Math. (2) 79 (1964), 59-103.

[12] Green H.S., A generalized method of field quantization, Phys. Rev. 90 (1953), 270-273.

[13] Lesimple M., Pinczon G., Deformations of the metaplectic representations, J. Math. Phys. 42 (2001), 18871899.

[14] Loday J.-L., Cyclic homology, Grundlehren der Mathematischen Wissenschaften, Vol. 301, Springer-Verlag, Berlin, 1992.

[15] McConnell J.C., Robson J.C., Noncommutative Noetherian rings. With the cooperation of L.W. Small, revised ed., Graduate Studies in Mathematics, Vol. 30, American Mathematical Society, Providence, RI, 2001. 
[16] Montgomery S., Constructing simple Lie superalgebras from associated graded algebras, J. Algebra 195 (1997), 558-579.

[17] Musson I.M., Some Lie superalgebras associated to the Weyl algebras, Proc. Amer. Math. Soc. 127 (1999), $2821-2827$.

[18] Palev T., Para-Bose and para-Fermi operators as generators of orthosymplectic Lie superalgebras, J. Math. Phys. 23 (1982), 1100-1102.

[19] Palev T., Algebraic structure of the Greens's ansatz and its q-deformed analogue, J. Phys. A: Math. Gen. 27 (1994), 7373-7385, hep-th/9406066.

[20] Pinczon G., The enveloping algebra of the Lie superalgebra osp(1,2), J. Algebra 132 (1990), 219-242.

[21] Pinczon G., On two theorems about symplectic reflection algebras, Lett. Math. Phys. 82 (2007), $237-253$.

[22] Pinczon G., Ushirobira R., New applications of graded Lie algebras to Lie algebras, generalized Lie algebras, and cohomology, J. Lie Theory 17 (2007), 633-667, math.RT/0507387.

[23] Pinczon G., Ushirobira R., Supertrace and superquadratic Lie structure on the Weyl algebra, and applications to formal inverse Weyl transform, Lett. Math. Phys. 74 (2005), 263-291, math.RT/0507092.

[24] Samelson H., Notes on Lie algebras, Van Nostrand Reinhold Mathematical Studies, no. 23, Van Nostrand Reinhold Co., New York - London - Melbourne, 1969.

[25] Scheunert M., The theory of Lie superalgebras. An introduction, Lecture Notes in Mathematics, Vol. 716, Springer-Verlag, Berlin, 1979.

[26] Sridharan R., Filtered algebras and representations of Lie algebras, Trans. Amer. Math. Soc. 100 (1961), $530-550$.

[27] Sternheimer D., Quantization is deformation, Contemp. Math. 391 (2005), 331-352.

[28] Wigner E.P., Do the equations of motion determine the quantum mechanical commutation relations?, Phys. Rev. 77 (1950), 711-712. 